\title{
ABOUT THE EULER-POINCARÉ CHARACTERISTIC OF SEMI-ALGEBRAIC SETS DEFINED WITH TWO INEQUALITIES
}

\author{
Nicolas DUTERTRE
}

\begin{abstract}
We express the Euler-Poincaré characteristic of a semi-algebraic set, which is the intersection of a non-singular complete intersection with two polynomial inequalities, in terms of the signatures of appropriate bilinear symmetric forms.
\end{abstract}

\section{Introduction}

Let $F=\left(F_{1}, \ldots, F_{k}\right): \mathbf{R}^{n} \rightarrow \mathbf{R}^{k}, n>k$, be a polynomial mapping such that $W_{\mathbf{R}}=F^{-1}(0)$ is a smooth non-empty manifold of dimension $n-k$. Let $g: \mathbf{R}^{n} \rightarrow \mathbf{R}$ be a polynomial. For $g=\omega=x_{1}^{2}+\cdots+x_{n}^{2}$, Szafraniec in [Sz2] defined a polynomial algebra $A_{\mathbf{R}}$ in terms of $F$ and $\omega$ and two bilimear symmetric forms $\Phi$ and $\Phi^{M}$ such that if $A_{\mathbf{R}}$ is finite dimensional and $\Phi^{M}$ is non-degenerate then

$$
\begin{aligned}
& \chi\left(W_{\mathbf{R}}\right)=(-1)^{k} \text { signature } \Phi \text { if } n-k \text { is odd, } \\
& \chi\left(W_{\mathbf{R}}\right)=\text { signature } \Phi^{M} \text { if } n-k \text { is even. }
\end{aligned}
$$

In [Dut1] we adapted his method to the case $g_{\mid W_{\mathbf{R}}}$ proper. We defined a polynomial algebra $A_{\mathbf{R}}$ in terms of $F$ and $g$ and four bilinear symmetric forms $\Phi, \Phi^{M}, \Phi_{g}$ and $\Phi_{g}^{M}$ such that if $A_{\mathbf{R}}$ is finite dimensional and $\Phi_{g}^{M}$ is non-degenerate then

- if $n-k$ is odd

$$
\begin{gathered}
\chi\left(W_{\mathbf{R}} \cap\{g \geq 0\}\right)-\chi\left(W_{\mathbf{R}} \cap\{g \leq 0\}\right)=(-1)^{k} \text { signature } \Phi, \\
\chi\left(W_{\mathbf{R}} \cap\{g \geq 0\}\right)+\chi\left(W_{\mathbf{R}} \cap\{g \leq 0\}\right)-2 \chi\left(W_{\mathbf{R}} \cap\{g=0\}\right)=(-1)^{k} \\
\text { signature } \Phi_{g},
\end{gathered}
$$

2000 Mathematics Subject Classification: 14P10, 14P25.

Servicio de Publicaciones. Universidad Complutense. Madrid, 2001 
- if $n-k$ is even

$$
\begin{aligned}
& \chi\left(W_{\mathbf{R}} \cap\{g \geq 0\}\right)-\chi\left(W_{\mathbf{R}} \cap\{g \leq 0\}\right)=\text { signature } \Phi_{g}^{M}, \\
& \chi\left(W_{\mathbf{R}} \cap\{g \geq 0\}\right)+\chi\left(W_{\mathbf{R}} \cap\{g \leq 0\}\right)=\text { signature } \Phi^{M} .
\end{aligned}
$$

The aim of this paper is to generalize these formulas in two ways. The first is to study the case where $g_{\mid W_{\mathbf{R}}}$ is not proper. For this we will define two polynomial algebras $A_{\mathbf{R}}$ and $B_{\mathbf{R}}$, four bilinear symmetric forms $\Phi, \Phi^{M}, \Phi_{g}, \Phi_{g}^{M}$ on $A_{\mathbf{R}}$ and two bilinear symmetric forms $\Psi$ and $\Psi_{\mu}$ on $B_{\mathbf{R}}$ such that if, $A_{\mathbf{R}}$ and $B_{\mathbf{R}}$ are finite dimensional and $\Phi_{g}$ is non-degenerate, then (see Theorem 4.4) :

- if $n-k$ is odd

$$
\begin{gathered}
\chi\left(W_{\mathbf{R}} \cap\{g \geq 0\}\right)+\chi\left(W_{\mathbf{R}} \cap\{g \leq 0\}\right)= \\
(-1)^{k}(\text { signature } \Phi-\text { signature } \Psi), \\
\chi\left(W_{\mathbf{R}} \cap\{g \geq 0\}\right)-\chi\left(W_{\mathbf{R}} \cap\{g \leq 0\}\right)= \\
(-1)^{k}\left(\text { signature } \Phi_{g}-\text { signature } \Psi_{\mu}\right),
\end{gathered}
$$

- if $n-k$ is even

$$
\begin{gathered}
\chi\left(W_{\mathbf{R}} \cap\{g \geq 0\}\right)+\chi\left(W_{\mathbf{R}} \cap\{g \leq 0\}\right)= \\
\text { signature } \Phi^{M}+(-1)^{k+1} \text { signature } \Psi, \\
\chi\left(W_{\mathbf{R}} \cap\{g \geq 0\}\right)-\chi\left(W_{\mathbf{R}} \cap\{g \leq 0\}\right)= \\
\text { signature } \Phi_{g}^{M}+(-1)^{k} \text { signature } \Psi_{\mu} .
\end{gathered}
$$

The second generalization will concern the following semi-algebraic sets :

$$
W_{\mathbf{R}} \cap\{g * 0, f ? 0\},
$$

where $*, ? \in\{\leq, \geq\}$ and $g, f: \mathbf{R}^{n} \rightarrow \mathbf{R}$ are polynomials. We will define three polynomials algebras $A_{\mathbf{R}}, B_{\mathbf{R}}$ and $C_{\mathbf{R}}$ and several bilinear symmetric forms on them. Under some conditions on the algebras and 
on the bilinear symmetric forms we will be able to express the following Euler characteristics

$$
\chi\left(W_{\mathbf{R}} \cap\{g * 0, f ? 0\}\right),
$$

in terms of signatures of suitable bilinear symmetric forms (see Theorem 5.1 and Theorem 6.1). As a consequence we will obtain formulas for the Euler characteristic of the semi-algebraic sets

$$
W_{\mathbf{R}} \cap\{g * 0\} \cap\{f=0\},
$$

where $W_{\mathbf{R}} \cap\{f=0\}$ admits some isolated singularities (see Corollary $6.2)$.

Remark 1.1. In [Dut1] we give formulas for $\chi\left(W_{\mathbf{R}} \cap\{g * 0, f ? 0\}\right)$ under a finite dimensional condition. But it is clear that this condition is not generic and holds only when $\operatorname{dim} W_{\mathbf{R}}=1$.

Finally we will study the case $\operatorname{dim} W_{\mathbf{R}}=2$ and we will show that in this case, we need only one polynomial algebra and thus we can obtain easier formulas.

Our main tools are Morse theory for manifolds with boundary, which is the subject of Section 2, and the theory of Frobenius algebras, which is the subject of Section 3. Section 4 is devoted to the study of the semialgebraic sets $W_{\mathbf{R}} \cap\{g * 0\}$ with $g_{\mid W_{\mathbf{R}}}$ non-proper. Section 5 and Section 6 are devoted to the sets $W_{\mathbf{R}} \cap\{g * 0, f ? 0\}$. In Section 7 , we study the case $\operatorname{dim} W_{\mathbf{R}}=2$. Our work relies on the machinery developed by Szafraniec in [Sz1] and [Sz2] and we will often refer to it.

The examples are computed with a program written by Andrzej Lecki. The author is grateful to him and Zbigniew Szafraniec for giving this programm and for explaining how to use it. He also thanks Karim Bekka for his comments on this paper.

\section{Morse theory for manifolds with boundary}

We recall the results of Morse theory for manifolds with boundary. Our reference is [HL] where the results are given for a $C^{\infty}$ manifold $M$ with boundary $\partial M$. For simplicity we will present the results for manifolds with boundary of type $M \cap\{g * 0\}, * \in\{\geq, \leq\}$, where $M$ is a $C^{\infty}$ manifold 
and $g: M \rightarrow \mathbf{R}$ a $C^{\infty}$ function such that $M \cap g^{-1}\{0\}$ is smooth. In fact this is the case we need in the following sections.

Let $M$ be a $C^{\infty}$ manifold of dimension $n$. Let $g: M \rightarrow \mathbf{R}$ be a $C^{\infty}$ function such that $\nabla g(x) \neq 0$ for all $x \in g^{-1}(0)$. This implies that $M \cap g^{-1}(0)$ is a smooth manifold of dimension $n-1$ and that $M \cap\{g \geq 0\}$ and $M \cap\{g \leq 0\}$ are smooth manifolds with boundary. Let $f: M \rightarrow \mathbf{R}$ be a smooth function. A critical point of $f_{\mid M \cap\{g \geq 0\}}$ (resp. $f_{\mid M \cap\{g \leq 0\}}$ ) is a critical point of $f_{\mid M \cap\{g>0\}}$ (resp. $f_{\mid M \cap\{g<0\}}$ ) or a critical point of $f_{\mid M \cap g^{-1}(0)}$.

Definition 2.1. Let $q \in M \cap g^{-1}(0)$. We say that $q$ is a correct critical point of $f_{\mid M \cap\{g \geq 0\}}$ (resp. $f_{\mid M \cap\{g \leq 0\}}$ ) if $q$ is a critical point of $f_{\mid M \cap g^{-1}(0)}$ and $q$ is not a critical point of $f_{\mid M}$.

We say that $q$ is a correct non-degenerate critical point of $f_{\mid M \cap\{g \geq 0\}}$ (resp. $f_{\mid M \cap\{g \leq 0\}}$ ) if $q$ is a correct critical point of $f_{\mid M \cap\{g \geq 0\}}$ (resp. $\left.f_{\mid M \cap\{g \leq 0\}}\right)$ and $q$ is a non-degenerate critical point of $f_{\mid M \cap g^{-1}(0)}$.

If $q$ is a correct critical point of $f_{\mid M \cap\{g \geq 0\}}$ (resp. $f_{\mid M \cap\{g \leq 0\}}$ ) then $\nabla f(q) \neq 0, \nabla f(q)$ and $\nabla g(q)$ are colinear and there is $\tau(q) \in \mathbf{R}^{*}$ with $\nabla f(q)=\tau(q) \cdot \nabla g(q)$.

Definition 2.2. If $q$ is a correct critical point of $f_{\mid M \cap\{g \geq 0\}}$ then

- $\nabla f(q)$ points inwards if and only if $\tau(q)>0$,

- $\nabla f(q)$ points outwards if and only if $\tau(q)<0$.

If $q$ is a correct critical point of $f_{\mid M \cap\{g \leq 0\}}$ then

- $\nabla f(q)$ points inwards if and only if $\tau(q)<0$,

- $\nabla f(q)$ points outwards if and only if $\tau(q)>0$.

Definition 2.3. A $C^{\infty}$ function $f: M \cap\{g \geq 0\} \rightarrow \mathbf{R}$ (resp. $M \cap\{g \leq$ $0\} \rightarrow \mathbf{R})$ is a correct function if all critical points of $f_{\mid M \cap g^{-1}(0)}$ are correct. A $C^{\infty}$ function $f: M \cap\{g \geq 0\} \rightarrow \mathbf{R}$ (resp. $M \cap\{g \leq 0\} \rightarrow$ $\mathbf{R}$ ) is a Morse correct function if $f_{\mid M \cap\{g>0\}}$ (resp. $f_{\mid M \cap\{g<0\}}$ ) admits only non-degenerate critical points and if $f$ admits only non-degenerate correct critical points.

Proposition 2.4. For any $C^{\infty}$ manifold $M$ and for any function $g: M \rightarrow \mathbf{R}$ such that $\nabla g(x) \neq 0$ for all $x \in g^{-1}(0)$, the set of $C^{\infty}$ 
functions $f: M \rightarrow \mathbf{R}$ such that $f_{\mid M \cap\{g \geq 0\}}$ and $f_{\mid M \cap\{g \leq 0\}}$ are Morse correct functions is dense in $C^{\infty}(M, \mathbf{R})$.

We will denote $\chi(M \cap\{g * 0\} \cap\{f ? 0\})$ by $\chi_{*, \text { ? }}$ and we will use the following result.

Theorem 2.5. Let $M$ be a $C^{\infty}$ manifold of dimension $n$ and let $g$ : $M \rightarrow \mathbf{R}$ be a $C^{\infty}$ function such that $\nabla g(x) \neq 0$ for all $x \in g^{-1}(0)$. Let $f: M \rightarrow \mathbf{R}$ be a $C^{\infty}$ function such that $f_{\mid M}$ is proper, and that $f_{\mid M \cap\{g \geq 0\}}$ and $f_{\mid M \cap\{g \leq 0\}}$ are Morse correct. Let $\left\{p_{i}\right\}$ be the set of critical points of $f_{\mid M}$ and $\left\{\lambda_{i}\right\}$ be the set of their respective indices. Let $\left\{q_{j}\right\}$ be the set of critical points of $f_{\mid M \cap g^{-1}(0)}$ and $\left\{\mu_{j}\right\}$ be the set of their respective indices. Then we have

$$
\begin{gathered}
\chi_{\geq, \geq}-\chi_{\geq,=}=\sum_{\substack{i / f\left(p_{i}\right)>0 \\
g\left(p_{i}\right)>0}}(-1)^{\lambda_{i}}+\sum_{\substack{j / f\left(q_{j}\right)>0 \\
\tau\left(q_{j}\right)>0}}(-1)^{\mu_{j}}, \\
\chi_{\geq, \leq-}-\chi_{\geq,=}=(-1)^{n} \sum_{\substack{i / f\left(p_{i}\right)<0 \\
g\left(p_{i}\right)>0}}(-1)^{\lambda_{i}}+(-1)^{n-1} \sum_{\substack{j / f\left(q_{j}\right)<0 \\
\tau\left(q_{j}\right)<0}}(-1)^{\mu_{j}},
\end{gathered}
$$

and

$$
\begin{gathered}
\chi_{\leq, \geq}-\chi_{\leq,=}=\sum_{\substack{i / f\left(p_{i}\right)>0 \\
g\left(p_{i}\right)<0}}(-1)^{\lambda_{i}}+\sum_{\substack{j / f\left(q_{j}\right)>0 \\
\tau\left(q_{j}\right)<0}}(-1)^{\mu_{j}}, \\
\chi_{\leq, \leq}-\chi_{\leq,=}=(-1)^{n} \sum_{\substack{i / f\left(p_{i}\right)<0 \\
g\left(p_{i}\right)<0}}(-1)^{\lambda_{i}}+(-1)^{n-1} \sum_{\substack{j / f\left(q_{j}\right)<0 \\
\tau\left(q_{j}\right)>0}}(-1)^{\mu_{j}} .
\end{gathered}
$$

\section{The global residue or Kronecker symbol}

In this section, we recall the construction of the global residue (or Kronecker symbol) on zero-dimensional polynomial algebras and we give its main properties. Actually we present Szafraniec's generalization [Sz2] of the global residue ([BCRS],[Ca],[Ku],[SS]).

Let $F=\left(f_{1}, \ldots, f_{N}\right): \mathbf{R}^{n} \rightarrow \mathbf{R}^{N}$, where $N \geq n$, be a polynomial mapping. We denote $\mathbf{R}\left[x_{1}, \ldots, x_{n}\right]$ by $\mathbf{R}[x]$. Let $A_{\mathbf{R}}=\frac{\mathbf{R}[x]}{(F)}$ and let us assume that $\operatorname{dim}_{\mathbf{R}} A_{\mathbf{R}}<+\infty, A_{\mathbf{R}}$ is in that case a zero-dimensional 
polynomial algebra (if $N=n$ it is a complete intersection). Let $V_{\mathbf{C}}$ (resp. $V_{\mathbf{R}}$ ) be the set of common zeros in $\mathbf{C}^{n}\left(\right.$ resp. $\mathbf{R}^{n}$ ) of $f_{1}, \ldots, f_{N}$; $V_{\mathbf{C}}$ is a finite set of points and we can write

$$
V_{\mathbf{C}}=\left\{p_{1}, \ldots, p_{m}\right\} \cup\left\{p_{m+1}, \overline{p_{m+1}}, \ldots, p_{s}, \overline{p_{s}}\right\},
$$

where

$$
V_{\mathbf{R}}=V_{\mathbf{C}} \cap \mathbf{R}^{n}=\left\{p_{1}, \ldots, p_{m}\right\},
$$

and $V_{\mathbf{C}} \backslash V_{\mathbf{R}}$ consists of pairs of conjuguate points.

We denote $A_{\mathbf{R}, p_{j}}$ (resp. $A_{\mathbf{C}, p_{j}}$ ) the local algebra $\mathcal{O}_{\mathbf{R}, p_{j}} /(F)$ (resp. $\left.\mathcal{O}_{\mathbf{C}, p_{j}} /(F)\right)$ where $\mathcal{O}_{\mathbf{R}, p_{j}}\left(\right.$ resp. $\mathcal{O}_{\mathbf{C}, p_{j}}$ ) is the ring of real (resp. complex) analytic germs at $p_{j}$.

Let $\Pi_{i}: A_{\mathbf{R}} \rightarrow A_{\mathbf{R}, p_{i}}, i=1, \ldots, m$, be the projection such that $\Pi_{i}(f)$ is the residue class of $f$ in $A_{\mathbf{R}, p_{i}}$. In the same way, we define $\Pi_{j}: A_{\mathbf{R}} \rightarrow A_{\mathbf{C}, p_{j}}, j=m+1, \ldots, s$. The natural projection

$$
\begin{aligned}
\Pi: A_{\mathbf{R}} & \rightarrow A_{\mathbf{R}, p_{1}} \times \cdots \times A_{\mathbf{R}, p_{m}} \times A_{\mathbf{C}, p_{m+1}} \times \cdots \times A_{\mathbf{C}, p_{s}} \\
f & \mapsto\left(\Pi_{1}(f), \ldots, \Pi_{m}(f), \Pi_{m+1}(f), \ldots, \Pi_{s}(f)\right)
\end{aligned}
$$

is an isomorphism of $\mathbf{R}$-algebras.

For $1 \leq i, j \leq n$, we define

$$
T_{i, j}(x, y)=\frac{f_{i}\left(y_{1}, \ldots, y_{j-1}, x_{j}, \ldots, x_{n}\right)-f_{i}\left(y_{1}, \ldots, y_{j}, x_{j+1}, \ldots, x_{n}\right)}{x_{j}-y_{j}} .
$$

It is easy to see that $T_{i, j}(x, y)$ defines a polynomial in $\mathbf{R}[x, y]$. We define a natural projection $\mathbf{R}[x, y] \rightarrow A_{\mathbf{R}} \otimes A_{\mathbf{R}}$ by

$$
x_{1}^{\alpha_{1}} \cdots x_{n}^{\alpha_{n}} y_{1}^{\beta_{1}} \cdots y_{n}^{\beta_{n}} \mapsto x_{1}^{\alpha_{1}} \cdots x_{n}^{\alpha_{n}} \otimes y_{1}^{\beta_{1}} \cdots y_{n}^{\beta_{n}} .
$$

Let $T$ be the image of $\operatorname{det}\left[T_{i, j}(x, y)\right]$ in $A_{\mathbf{R}} \otimes A_{\mathbf{R}}$. Let $d=\operatorname{dim}_{\mathbf{R}} A_{\mathbf{R}}$ and let $e_{1}, \ldots, e_{d}$ be a basis in $A_{\mathbf{R}}$. Then $\operatorname{dim}_{\mathbf{R}} A_{\mathbf{R}} \otimes A_{\mathbf{R}}=d^{2}$ and the $e_{i} \otimes e_{j}, 1 \leq i, j \leq d$, form a basis in $A_{\mathbf{R}} \otimes A_{\mathbf{R}}$. Thus there exist $t_{i j} \in \mathbf{R}$ such that

$$
T=\sum_{i, j=1}^{d} t_{i j} e_{i} \otimes e_{j}=\sum_{i=1}^{d} e_{i} \otimes \hat{e}_{i},
$$

where $\hat{e}_{i}=\sum_{j=1}^{d} t_{i j} e_{j}$. 
Theorem 3.1. Assume that for each $p \in V_{\mathbf{C}},\left(f_{1}, \ldots, f_{N}\right)=\left(f_{1}, \ldots, f_{n}\right)$ in $A_{\mathbf{C}, p}$. Then $\hat{e}_{1}, \ldots \hat{e}_{d}$ form a basis in $A_{\mathbf{R}}$.

Proof. See [Sz2] p353-354.

Hence we can find $a_{1}, \ldots, a_{d}$ in $\mathbf{R}$ such that $1=a_{1} \hat{e}_{1}+\cdots+a_{d} \hat{e}_{d}$ in $A_{\mathbf{R}}$. We define a linear functional $\phi: A_{\mathbf{R}} \rightarrow \mathbf{R}$ in the following way

$$
\phi(g)=a_{1} b_{1}+\cdots+a_{d} b_{d} \text { if } g=b_{1} e_{1}+\cdots+b_{d} e_{d} \text { in } A_{\mathbf{R}} .
$$

For all $1 \leq i \leq s$, let $\eta_{i}: A_{\mathbf{K}, p_{i}} \rightarrow A_{\mathbf{R}}$ denote the restriction of $\Pi^{-1}$ to

$$
\{0\} \times \cdots \times A_{\mathbf{K}, p_{i}} \times \cdots \times\{0\},
$$

where $\mathbf{K}=\mathbf{R}$ or $\mathbf{C}$ and let $\phi_{i}=\phi \circ \eta_{i}$ be the natural restriction of $\phi$ to $A_{\mathbf{K}, p_{i}}$. Let

$$
h(x)=\frac{\partial\left(f_{1}, \ldots, f_{n}\right)}{\partial\left(x_{1}, \ldots, x_{n}\right)}(x),
$$

write $h_{i}=\Pi_{i}(\bar{h})$ where $\bar{h}$ is the image in $A_{\mathbf{R}}$ of $h$. Then we have

Theorem 3.2. Assume that for each $p \in V_{\mathbf{C}},\left(f_{1}, \ldots, f_{N}\right)=\left(f_{1}, \ldots, f_{n}\right)$ in $A_{\mathbf{C}, p}$. Then for each $i \in\{1, \ldots, n\}, \phi_{i}\left(h_{i}\right)=\operatorname{dim}_{\mathbf{K}} A_{\mathbf{K}, p_{i}}$. In particular, for each $i \in\{1, \ldots, s\}, \phi_{i}\left(h_{i}\right)>0$.

Proof. See [Sz2] p 353-354.

Remark 3.3. When $N=n$ it is clear that the assumption holds. In that case, $\phi$ is the usual global residue ([BCRS], $[\mathrm{Ca}],[\mathrm{Ku}],[\mathrm{SS}])$.

Let $u \in \mathbf{R}\left[x_{1}, \ldots, x_{n}\right]$ and let us define the following bilinear symmetric form $\Phi_{u}$ :

$$
\Phi_{u}: A_{\mathbf{R}} \times A_{\mathbf{R}} \rightarrow \mathbf{R} \text { defined by } \Phi_{u}\left(g_{1}, g_{2}\right)=\phi\left(u g_{1} g_{2}\right) .
$$

We have

Theorem 3.4. $\Phi_{u}$ is non-degenerate if and only if for each $p \in V_{\mathbf{C}}$, $u(p) \neq 0$.

Proof. See [Sz2] p353 and [Sz3] p304. 
For all $1 \leq j \leq m$, let $\Phi_{u}^{j}$ be the bilinear symmetric form defined on $A_{\mathbf{R}, p_{j}}$ by $\Phi_{u}^{j}\left(g_{1}, g_{2}\right)=\phi_{j}\left(u g_{1} g_{2}\right)$. Then

\section{Proposition 3.5.}

$$
\text { signature } \Phi_{u}=\sum_{j=1}^{m} \text { signature } \Phi_{u}^{j} .
$$

Proof. It is clear.

Now we investigate the case $\Phi_{u}$ degenerate. Let $d=\operatorname{dim}_{\mathbf{R}} A_{\mathbf{R}}$. For $e \geq d$, let $\Phi_{u^{e}}$ be the bilinear symmetric form defined on $A_{\mathbf{R}}$ by

$$
\Phi_{u^{e}}\left(g_{1}, g_{2}\right)=\Phi\left(u^{e} g_{1} g_{2}\right),
$$

and let $\Phi_{u^{e}}^{j}$ be the natural restriction of $\Phi_{u^{e}}$ to $A_{\mathbf{R}, p_{j}}$. We have

Proposition 3.6. If $\Phi_{u}$ is degenerate then there exists $p \in V_{\mathbf{C}}$ such that $u(p)=0$ and

signature $\Phi_{u^{e}}=\sum$ signature $\Phi_{u^{e}}^{j}$ where $1 \leq j \leq m$ and $u\left(p_{j}\right) \neq 0$.

Proof. See [Dut1] Proposition 4.1 or [Dut2] Proposition 2.7.

\section{Study of the semi-algebraic sets $W_{\mathbf{R}} \cap\{g \geq 0\}$ and $W_{\mathbf{R}} \cap\{g \leq 0\}$}

Let $F:\left(F_{1}, \ldots, F_{k}\right): \mathbf{R}^{n} \rightarrow \mathbf{R}^{k}, n>k$, be a polynomial mapping such that $W_{\mathbf{C}}=\left\{x \in \mathbf{C}^{n} / F(x)=0\right\}$ is a smooth complex manifold of dimension $n-k$, which implies that $W_{\mathbf{R}}=\left\{x \in \mathbf{R}^{n} / F(x)=0\right\}$ is a smooth real manifold of dimension $n-k$, provided it is not empty. Let

$$
M=\frac{\partial\left(F_{1}, \ldots, F_{k}\right)}{\partial\left(x_{1}, \ldots, x_{k}\right)} .
$$


Let $\omega=x_{1}^{2}+\ldots+x_{n}^{2}$, let $I$ be the ideal generated by $F_{1}, \ldots, F_{k}$ and all $(k+1) \times(k+1)$ minors

$$
\frac{\partial\left(\omega, F_{1}, \ldots, F_{k}\right)}{\partial\left(x_{i_{1}}, \ldots, x_{i_{k+1}}\right)}
$$

Let $A_{\mathbf{R}}=\frac{\mathbf{R}[x]}{I}$ and $V_{\mathbf{C}}=\left\{p \in \mathbf{C}^{n} /\right.$ for all $\left.u \in I, u(p)=0\right\}$. Assume that $\operatorname{dim}_{\mathbf{R}} A_{\mathbf{R}}<+\infty$, hence $V_{\mathbf{C}}$ is finite and

$$
V_{\mathbf{C}}=\left\{p_{1}, \ldots, p_{m}\right\} \cup\left\{p_{m+1}, \overline{p_{m+1}}, \ldots, p_{s}, \overline{p_{s}}\right\} .
$$

The set of critical points of $\omega_{\mid W_{\mathbf{C}}}$ is $V_{\mathbf{C}}$ and $V_{\mathbf{R}}=V_{\mathbf{C}} \cap \mathbf{R}^{n}=\left\{p_{1}, \ldots, p_{m}\right\}$ is the set of critical points of $\omega_{\mid W_{\mathbf{R}}}$. After an appropriate change of coordinates, one may assume that for each $p \in V_{\mathbf{C}}, M(p) \neq 0$.

Now let $g: \mathbf{R}^{n} \rightarrow \mathbf{R}$ be a polynomial such that $g^{-1}(0) \cap W_{\mathbf{R}}$ is a smooth manifold of dimension $n-k-1$. Let $\left(x_{1}, \ldots, x_{n} ; \lambda_{1}, \ldots, \lambda_{k} ; \mu\right)$ be a coordinate system in $\mathbf{R}^{n+k+1}$ and let

$$
\begin{array}{ccc}
H: \mathbf{R}^{n+k+1} & \rightarrow & \mathbf{R}^{n+k+1} \\
\left(x_{1}, \ldots, x_{n} ; \lambda_{1}, \ldots, \lambda_{k}, \mu\right) & \mapsto\left(\nabla \omega+\sum_{i=1}^{k} \lambda_{i} \nabla F_{i}+\mu \nabla g ; F_{1}, \ldots, F_{k}, g\right) .
\end{array}
$$

Let $B_{\mathbf{R}}=\mathbf{R}[x, \lambda, \mu] /(H)$ and assume that $\operatorname{dim}_{\mathbf{R}} B_{\mathbf{R}}<+\infty$. Let

$$
Y_{\mathbf{R}}=\left\{(q, \lambda, \mu) \in \mathbf{R}^{n+k+1} / H(q, \lambda, \mu)=0\right\} .
$$

Then $Y_{\mathbf{R}}$ is a finite set of points and we write

$$
Y_{\mathbf{R}}=\left\{\left(q_{1}, \lambda_{1}, \mu_{1}\right), \ldots,\left(q_{l}, \lambda_{l}, \mu_{l}\right)\right\} .
$$

The points $q_{1}, \ldots, q_{l}$ are exactly the critical points of $\omega_{\mid W_{\mathbf{R}} \cap g^{-1}(0)}$ (see [Sz1]).

\subsection{Two local studies}

We investigate the situation at a critical point of $\omega_{\mid W_{\mathbf{C}}}$ and at a critical point of $\omega_{\mid W_{\mathbf{R}} \cap g^{-1}(0)}$. We begin with $\omega_{\mid W_{\mathbf{C}}}$.

For all $p \in V_{\mathbf{K}}(\mathbf{K}=\mathbf{R}$ or $\mathbf{C}), \mathcal{O}_{\mathbf{K}, p}$ is the ring of analytic function germs defined near $p$. We set

$$
m_{j}(x)=\frac{\partial\left(\omega, F_{1}, \ldots, F_{k}\right)}{\partial\left(x_{1}, \ldots, x_{k} ; x_{j}\right)} \quad \text { for each } j \geq k+1
$$




$$
h(x)=\frac{\partial\left(F_{1}, \ldots, F_{k} ; m_{k+1}, \ldots, m_{n}\right)}{\partial\left(x_{1}, \ldots, x_{n}\right)} .
$$

Let $\left(F_{1}, \ldots, F_{k}\right)$ be the ideal generated by $F_{1}, \ldots, F_{k}$ in $\mathcal{O}_{\mathbf{K}, p}$, let $I_{\mathbf{K}, p}$ be the one generated by $F_{1}, \ldots, F_{k}$ and all $(k+1) \times(k+1)$ minors

$$
\frac{\partial\left(\omega, F_{1}, \ldots, F_{k}\right)}{\partial\left(x_{i_{1}}, \ldots, x_{i_{k+1}}\right)}
$$

and $J_{\mathbf{K}, p}$ the one generated by $F_{1}, \ldots, F_{k}, m_{k+1}, \ldots, m_{n}$. Clearly $\left(F_{1}, \ldots, F_{k}\right) \subset J_{\mathbf{K}, p} \subset I_{\mathbf{K}, p}$. Let $A_{\mathbf{K}, p}=\mathcal{O}_{\mathbf{K}, p} / I_{\mathbf{K}, p}$. Since $\operatorname{dim}_{\mathbf{R}} A_{\mathbf{R}}<+\infty$, we have that for each $p \in V_{\mathbf{K}}$, $\operatorname{dim}_{\mathbf{K}} A_{\mathbf{K}, p}<+\infty$ and then

Lemma 4.1. for each $p \in V_{\mathbf{K}}, I_{\mathbf{K}, p}=J_{\mathbf{K}, p}$.

Proof. See [Sz2,p349-350] or [Dut3] appendix.

Now we study the local situation at a point $p_{j} \in V_{\mathbf{R}}$. We have $M\left(p_{j}\right) \neq 0$ and $\operatorname{dim}_{\mathbf{R}} A_{\mathbf{R}, p_{j}}<+\infty$. Let $\phi: A_{\mathbf{R}, p_{j}} \rightarrow \mathbf{R}$ be a linear functional such that $\phi(h)>0$. Let $u \in \mathcal{O}_{\mathbf{R}, p_{j}}$ be a real analytic germ. Let $\Phi_{u}^{j}$ (resp. $\Phi_{u}^{M, j}$ ) be the bilinear symmetric form on $A_{\mathbf{R}, p_{j}}$ given by $\Phi_{u}^{j}\left(g_{1}, g_{2}\right)=\phi\left(u g_{1} g_{2}\right)\left(\operatorname{resp} . \Phi_{u}^{M, j}\left(g_{1}, g_{2}\right)=\phi\left(M u g_{1} g_{2}\right)\right)$. Let $\tilde{\omega}: W_{\mathbf{R}} \rightarrow$ $\mathbf{R}$ be a Morse function which uniformly approximates $\omega_{\mid W_{\mathbf{R}}}$ in the $C^{2}$ topology. Let $\left\{p_{j i}\right\}$ be the set of Morse critical points of $\tilde{\omega}$ lying near $p_{j}$ and let $\left\{\lambda_{j i}\right\}$ be the set of their respective indices. The following proposition is an easy generalization of Proposition 3.5, p352 [Sz2].

\section{Proposition 4.2.}

1. $\Phi_{u}^{j}$ is non-degenerate if and only if $u\left(p_{j}\right) \neq 0$,

2. $\sum_{i}(-1)^{\lambda_{j i}}=(-1)^{k} \operatorname{sign} u\left(p_{j}\right) \cdot$ signature $\Phi_{u}^{j}$ if $n-k$ is odd,

3. $\sum_{i}(-1)^{\lambda_{j i}}=\operatorname{sign} u\left(p_{j}\right) \cdot$ signature $\Phi_{u}^{M, j}$ if $n-k$ is even.

Now we study the situation at a critical point $q_{j}$ of $\omega_{\mid W_{\mathbf{R}} \cap g^{-1}(0)}$. Let $\psi: B_{\mathbf{R}, q_{j}} \rightarrow \mathbf{R}$ be a linear functional such that $\psi(\operatorname{Jac} H)>0$ 
where $B_{\mathbf{R}, q_{j}}=\mathcal{O}_{\mathbf{R},\left(q_{j}, \lambda_{j}, \mu_{j}\right)} /(H)$ and let $v \in \mathcal{O}_{\mathbf{R},\left(q_{j}, \lambda_{j}, \mu_{j}\right)}$ be a real analytic germ. Let $\Psi_{v}^{j}$ be the bilinear symmetric form on $B_{\mathbf{R}, q_{j}}$ given by $\Psi_{v}^{j}\left(g_{1}, g_{2}\right)=\psi\left(v g_{1} g_{2}\right)$. Let $\tilde{\tilde{\omega}}: W_{\mathbf{R}} \cap g^{-1}(0) \rightarrow \mathbf{R}$ be a Morse function which uniformly approximates $\omega_{\mid W_{\mathbf{R}} \cap g^{-1}(0)}$ in the $C^{2}$-topology. Let $\left\{q_{j i}\right\}$ be the set of Morse critical points of $\tilde{\tilde{\omega}}$ lying near $q_{j}$ and let $\left\{\mu_{j i}\right\}$ be the set of their respective indices. Then we have

\section{Proposition 4.3.}

1. $\Psi_{v}^{j}$ is non-degenerate if and only if $v\left(q_{j}, \lambda_{j}, \mu_{j}\right) \neq 0$.

2. In that case $\sum_{i}(-1)^{\mu_{j i}}=(-1)^{k+1} \operatorname{sign} v\left(q_{j}, \lambda_{j}, \mu_{j}\right) \cdot$ signature $\Psi_{v}^{j}$.

Proof. The first part is proved in [Sz2] Lemma 2.2. For the second point, we use [Sz1] Lemma 1.4 and the Eisenbud-Levine formula (see $[\mathrm{AGV}],[\mathrm{Ei}],[\mathrm{EL}])$.

\subsection{Global study}

Recall that $A_{\mathbf{R}}=\mathbf{R}[x] / I$ is finite dimensional, $V_{\mathbf{C}}=\left\{p \in \mathbf{C}^{n} /\right.$ for all $u \in$ $I u(p)=0\}$ is the set of critical points of $\omega_{\mid W_{\mathbf{C}}}$ and $V_{\mathbf{R}}=\left\{p_{1}, \ldots, p_{m}\right\}$. Since one can assume that for all $p \in V_{\mathbf{C}}, M(p) \neq 0$ then, from Section 3 and the above Lemma 4.1, we can consider the global residue $\phi$ on $A_{\mathbf{R}}$. With this global residue, we construct the following bilinear symmetric forms on $A_{\mathbf{R}}$ :

$$
\begin{array}{ccccc}
\Phi & : & A_{\mathbf{R}} \times A_{\mathbf{R}} \rightarrow \mathbf{R} & \text { defined by } & \Phi\left(g_{1}, g_{2}\right)=\phi\left(g_{1} g_{2}\right), \\
\Phi_{g} & : & A_{\mathbf{R}} \times A_{\mathbf{R}} \rightarrow \mathbf{R} & \text { defined by } & \Phi_{g}\left(g_{1}, g_{2}\right)=\phi\left(g g_{1} g_{2}\right), \\
\Phi^{M} & : & A_{\mathbf{R}} \times A_{\mathbf{R}} \rightarrow \mathbf{R} & \text { defined by } & \Phi^{M}\left(g_{1}, g_{2}\right)=\phi\left(M g_{1} g_{2}\right), \\
\Phi_{g}^{M} & : & A_{\mathbf{R}} \times A_{\mathbf{R}} \rightarrow \mathbf{R} & \text { defined by } & \Phi_{g}^{M}\left(g_{1}, g_{2}\right)=\phi\left(M g g_{1} g_{2}\right) .
\end{array}
$$

Since $B_{\mathbf{R}}=\mathbf{R}[x] /(H)$ is finite dimensional, we can consider the global residue $\psi$ on $B_{\mathbf{R}}$ and we can construct the following bilinear symmetric forms on $B_{\mathbf{R}}$ :

$$
\begin{array}{ccccc}
\Psi & : & B_{\mathbf{R}} \times B_{\mathbf{R}} \rightarrow \mathbf{R} & \text { defined by } & \Psi\left(g_{1}, g_{2}\right)=\psi\left(g_{1} g_{2}\right), \\
\Psi_{\mu} & : & B_{\mathbf{R}} \times B_{\mathbf{R}} \rightarrow \mathbf{R} & \text { defined by } & \Psi_{\mu}\left(g_{1}, g_{2}\right)=\psi\left(\mu g_{1} g_{2}\right) .
\end{array}
$$


Recall that $Y_{\mathbf{R}}=\left\{\left(q_{1}, \lambda_{1}, \mu_{1}\right), \ldots,\left(q_{l}, \lambda_{l}, \mu_{l}\right)\right\}$. We will denote $W_{\mathbf{R}} \cap\{g * 0\}$ by $W_{\mathbf{R}}(g * 0)$ where $* \in\{\leq,=, \geq\}$.

Theorem 4.4. Assume the following conditions

- $W_{\mathbf{C}}$ is a smooth complex manifold of dimension $n-k$ and $W_{\mathbf{R}}$ is non-empty,

- $W_{\mathbf{C}} \cap g_{\mathbf{C}}^{-1}(0)$ is a smooth complex manifold of dimension $n-k-1$ and $W_{\mathbf{R}} \cap g^{-1}(0)$ is non-empty,

- for each $p \in V_{\mathbf{C}}, M(p) \neq 0$,

- $\Phi_{g}$ is non-degenerate,

then

1. $W_{\mathbf{R}}$ is a smooth real manifold of dimension $n-k$,

2. $W_{\mathbf{R}} \cap g^{-1}(0)$ is a smooth real manifold of dimension $n-k-1$,

3. $\Phi, \Phi^{M}, \Phi_{g}^{M}$ and $\Psi$ are non-degenerate,

4. $\Psi_{\mu}$ is non-degenerate,

5. All critical points of $\omega_{\mid W_{\mathbf{R}}(g \geq 0)}$ and of $\omega_{\mid W_{\mathbf{R}}(g \leq 0)}$ lying in $W_{\mathbf{R}} \cap$ $g^{-1}(0)$ are correct,

6. if $n-k$ is odd

$$
\begin{gathered}
\chi\left(W_{\mathbf{R}}(g \geq 0)\right)+\chi\left(W_{\mathbf{R}}(g \leq 0)\right)= \\
(-1)^{k}(\text { signature } \Phi-\text { signature } \Psi), \\
\chi\left(W_{\mathbf{R}}(g \geq 0)\right)-\chi\left(W_{\mathbf{R}}(g \leq 0)\right)= \\
(-1)^{k}\left(\text { signature } \Phi_{g}-\text { signature } \Psi_{\mu}\right),
\end{gathered}
$$

7. if $n-k$ is even

$$
\chi\left(W_{\mathbf{R}}(g \geq 0)\right)+\chi\left(W_{\mathbf{R}}(g \leq 0)\right)=
$$

signature $\Phi^{M}+(-1)^{k+1}$ signature $\Psi$, $\chi\left(W_{\mathbf{R}}(g \geq 0)\right)-\chi\left(W_{\mathbf{R}}(g \leq 0)\right)=$ signature $\Phi_{g}^{M}+(-1)^{k}$ signature $\Psi_{\mu}$. 
Proof. 1. and 2. are clear.

3. is an application of Theorem 3.4.

Since $\Phi_{g}$ is non-degenerate, for all $p \in V_{\mathbf{C}}, g(p) \neq 0$. This means that there is no critical point of $\omega_{\mid W_{\mathbf{C}}}$ in the zero locus of $g$. Thus for every point $(q, \lambda, \mu) \in \mathbf{C}^{n+k+1}$ such that $H(q, \lambda, \mu)=0, \mu \neq 0$ which implies that $\Psi_{\mu}$ is non-degenerate and that the critical points of $\omega_{\mid W_{\mathbf{R}} \cap\{g \geq 0\}}$ and $\omega_{\mid W_{\mathbf{R}} \cap\{g \leq 0\}}$ lying in $g^{-1}(0)$ are correct. This proves $\mathbf{4}$. and $\mathbf{5}$.

To show 6., we choose a function $\tilde{\omega}: W_{\mathbf{R}} \rightarrow \mathbf{R}$ which approximates $\omega_{\mid W_{\mathbf{R}}}$ such that $\tilde{\omega}_{\mid W_{\mathbf{R}} \cap\{g \geq 0\}}$ and $\tilde{\omega}_{\mid W_{\mathbf{R}} \cap\{g \leq 0\}}$ are Morse correct functions. For all $j \in\{1, \ldots, m\}$, let $\left\{p_{j 1}, \ldots, p_{j \sigma(j)}\right\}$ be the set of critical points of $\tilde{\omega}_{\mid W_{\mathbf{R}}}$ lying near $p_{j}$ and let $\left\{\lambda_{j 1}, \ldots, \lambda_{j \sigma(j)}\right\}$ be the set of their respective indices. For all $s \in\{1, \ldots, l\}$, let $\left\{q_{s 1}, \ldots, q_{s \tau(s)}\right\}$ be the set of critical points of $\tilde{\omega}_{\mid W_{\mathbf{R}} \cap g^{-1}(0)}$ lying near $q_{s}$ and let $\left\{\rho_{s 1}, \ldots, \rho_{s \tau(s)}\right\}$ be the set of their respective indices. Applying Theorem 2.5, we have

$$
\begin{aligned}
& \chi\left(W_{\mathbf{R}}(g \geq 0)\right)=\sum_{j / g\left(p_{j}\right)>0} \sum_{i=1}^{\sigma(j)}(-1)^{\lambda_{j i}}+\sum_{s / \mu_{s}<0} \sum_{i=1}^{\tau(s)}(-1)^{\rho_{s i}}, \\
& \chi\left(W_{\mathbf{R}}(g \leq 0)\right)=\sum_{j / g\left(p_{j}\right)<0} \sum_{i=1}^{\sigma(j)}(-1)^{\lambda_{j i}}+\sum_{s / \mu_{s}>0} \sum_{i=1}^{\tau(s)}(-1)^{\rho_{s i}} .
\end{aligned}
$$

Combining these two equalities gives

$$
\begin{aligned}
& \chi\left(W_{\mathbf{R}}(g \geq 0)\right)+\chi\left(W_{\mathbf{R}}(g \leq 0)\right)=\sum_{j} \sum_{i=1}^{\sigma(j)}(-1)^{\lambda_{j i}}+\sum_{s} \sum_{i=1}^{\tau(s)}(-1)^{\rho_{s i}}, \\
& \chi\left(W_{\mathbf{R}}(g \geq 0)\right)-\chi\left(W_{\mathbf{R}}(g \leq 0)\right)=\sum_{j} \operatorname{sign} g\left(p_{j}\right) \sum_{i=1}^{\sigma(j)}(-1)^{\lambda_{j i}}- \\
& \sum_{s} \operatorname{sign} \mu_{s} \sum_{i=1}^{\tau(s)}(-1)^{\rho_{s i}} .
\end{aligned}
$$

Using Proposition 4.2 and Proposition 4.3 and assuming $n-k$ odd, we get

$$
\begin{aligned}
& \chi\left(W_{\mathbf{R}}(g \geq 0)\right)+\chi\left(W_{\mathbf{R}}(g \leq 0)\right)= \\
& (-1)^{k} \sum_{j} \text { signature } \Phi^{j}+(-1)^{k+1} \sum_{s} \text { signature } \Psi^{s},
\end{aligned}
$$




$$
\begin{aligned}
& \chi\left(W_{\mathbf{R}}(g \geq 0)\right)-\chi\left(W_{\mathbf{R}}(g \leq 0)\right)= \\
& (-1)^{k} \sum_{j} \text { signature } \Phi_{g}^{j}+(-1)^{k} \sum_{s} \text { signature } \Psi_{\mu}^{s} .
\end{aligned}
$$

Hence, by Proposition 3.5

$\chi\left(W_{\mathbf{R}}(g \geq 0)\right)+\chi\left(W_{\mathbf{R}}(g \leq 0)\right)=(-1)^{k}($ signature $\Phi-$ signature $\Psi)$,

$\chi\left(W_{\mathbf{R}}(g \geq 0)\right)-\chi\left(W_{\mathbf{R}}(g \leq 0)\right)=(-1)^{k}\left(\right.$ signature $\Phi_{g}+$ signature $\left.\Psi_{\mu}\right)$.

We prove the case $n-k$ even in a similar way.

Corollary 4.5. Under the same assumptions, we have

- If $n-k$ is odd

$$
\begin{aligned}
\chi\left(W_{\mathbf{R}}(g \geq 0)\right)= & \frac{1}{2}(-1)^{k}\left(\text { signature } \Phi+\text { signature } \Phi_{g}\right. \\
& \text { - } \left.\text { signature } \Psi+\text { signature } \Psi_{\mu}\right), \\
\chi\left(W_{\mathbf{R}}(g \leq 0)\right)= & \frac{1}{2}(-1)^{k}\left(\text { signature } \Phi-\text { signature } \Phi_{g}\right. \\
& \text { - } \left.\text { signature } \Psi-\text { signature } \Psi_{\mu}\right) .
\end{aligned}
$$

- If $n-k$ is even

$$
\begin{gathered}
\chi\left(W_{\mathbf{R}}(g \geq 0)\right)=\frac{1}{2}\left(\text { signature } \Phi^{M}+\text { signature } \Phi_{g}^{M}\right) \\
+\frac{1}{2}(-1)^{k}\left(\text { signature } \Psi-\text { signature } \Psi_{\mu}\right), \\
\chi\left(W_{\mathbf{R}}(g \leq 0)\right)=\frac{1}{2}\left(\text { signature } \Phi^{M}-\text { signature } \Phi_{g}^{M}\right) \\
+\frac{1}{2}(-1)^{k}\left(\text { signature } \Psi+\text { signature } \Psi_{\mu}\right) .
\end{gathered}
$$

Proof. It is clear. 


\subsection{Examples}

Example 1. The first example is trivial but it enables us to check our formulas. Let $W_{\mathbf{R}}=\mathbf{R}^{2}$ and let $g\left(x_{1}, x_{2}\right)=x_{1}-1$. We are in the situation $n=2$ and $k=0$. The corresponding algebras are

$$
A_{\mathbf{R}}=\frac{\mathbf{R}\left[x_{1}, x_{2}\right]}{\left(2 x_{1}, 2 x_{2}\right)} \text { and } B_{\mathbf{R}}=\frac{\mathbf{R}\left[x_{1}, x_{2}, \mu\right]}{\left(2 x_{1}+\mu, 2 x_{2}, x_{1}-1\right)}
$$

The computer gives

- $\operatorname{dim}_{\mathbf{R}} A_{\mathbf{R}}=1$, signature $\Phi=1, \operatorname{rank} \Phi_{g}=1$ and signature $\Phi_{g}=$ -1 ,

- $\operatorname{dim}_{\mathbf{R}} B_{\mathbf{R}}=1$, signature $\Psi=-1$, signature $\Psi_{\mu}=-1$ and rank $\Psi_{\mu}=1$,

so, applying Theorem 4.4, we find

$$
\begin{aligned}
& \chi\left(x_{1} \geq 1\right)+\chi\left(x_{1} \leq 1\right)=2, \\
& \chi\left(x_{1} \geq 1\right)-\chi\left(x_{1} \leq 1\right)=0 .
\end{aligned}
$$

Example 2. Let $W_{\mathbf{R}}=\mathbf{R}^{2}$ and let $g=x_{2}^{5}+x_{1}^{2} x_{2}^{2}-x_{2}+1$. Computations give

- $\operatorname{dim}_{\mathbf{R}} A_{\mathbf{R}}=1$, signature $\Phi=1$, signature $\Phi_{g}=1$ and $\operatorname{rank} \Phi_{g}=1$,

- $\operatorname{dim}_{\mathbf{R}} B_{\mathbf{R}}=7$, signature $\Psi=-1$, signature $\Psi_{\mu}=-1$ and rank $\Psi_{\mu}=7$.

so, applying Theorem 4.4, we find

$$
\begin{aligned}
& \chi(g \geq 0)+\chi(g \leq 0)=2, \\
& \chi(g \geq 0)-\chi(g \leq 0)=0 .
\end{aligned}
$$

Example 3. Let $W_{\mathbf{R}}=\mathbf{R}^{3}$ and let $g=x_{1}^{3}+x_{2} x_{3}+x_{3}^{2}-1$. The computer gives

- $\operatorname{dim}_{\mathbf{R}} A_{\mathbf{R}}=1$, signature $\Phi=1$, signature $\Phi_{g}=-1$ and rank $\Phi_{g}=1$, 
- $\operatorname{dim}_{\mathbf{R}} B_{\mathbf{R}}=11$, signature $\Psi=-1$, signature $\Psi_{\mu}=1$ and rank $\Psi_{\mu}=11$.

so, applying Theorem 4.4, we find

$$
\begin{gathered}
\chi(g \geq 0)+\chi(g \leq 0)=2, \\
\chi(g \geq 0)-\chi(g \leq 0)=-2 .
\end{gathered}
$$

\section{Study of the semi-algebraic sets defined with two inequalities}

In this section we are interested in computing the Euler characteristic of semi-algebraic sets defined with two inequalities. For convenience we will denote $W_{\mathbf{R}} \cap\{g * 0\} \cap\{f ? 0\}$ by $W_{\mathbf{R}}(g * 0, f ? 0)$ and $\chi\left(W_{\mathbf{R}}(g * 0, f ? 0)\right)$ by $\chi_{*, ?}$ where $*, ? \in\{\leq,=, \geq\}$. We will proceed as in the previous section, replacing $\omega$ by a polynomial $f$ such that $f_{\mid W_{\mathbf{R}}}$ is proper.

Let $F=\left(F_{1}, \ldots, F_{k}\right): \mathbf{R}^{n} \rightarrow \mathbf{R}^{k}, n>k$, be a polynomial mapping such that $W_{\mathbf{C}}=\left\{x \in \mathbf{C}^{n} / F(x)=0\right\}$ is a smooth complex manifold of dimension $n-k$, which implies that $W_{\mathbf{R}}=\left\{x \in \mathbf{R}^{n} / F(x)=0\right\}$ is a smooth real manifold of dimension $n-k$, provided it is not empty. Let

$$
M=\frac{\partial\left(F_{1}, \ldots, F_{k}\right)}{\partial\left(x_{1}, \ldots, x_{k}\right)} .
$$

Let $g: \mathbf{R}^{n} \rightarrow \mathbf{R}$ be a polynomial such that $W_{\mathbf{R}} \cap g^{-1}(0)$ is a smooth manifold of dimension $n-k-1$. Let $f: \mathbf{R}^{n} \rightarrow \mathbf{R}$ be a polynomial, let $I$ be the ideal generated by $F_{1}, \ldots, F_{k}$ and all $(k+1) \times(k+1)$ minors $\frac{\partial\left(f, F_{1}, \ldots, F_{k}\right)}{\partial\left(x_{i_{1}}, \ldots, x_{i_{k+1}}\right)}$. Let $A_{\mathbf{R}}=\mathbf{R}[x] / I$ and $V_{\mathbf{C}}=\left\{p \in \mathbf{C}^{n} /\right.$ for all $u \in$ $I u(p)=0\}$. Assume that $\operatorname{dim}_{\mathbf{R}} A_{\mathbf{R}}<+\infty$, hence $V_{\mathbf{C}}$ is finite and

$$
V_{\mathbf{C}}=\left\{p_{1}, \ldots, p_{m}\right\} \cup\left\{p_{m+1}, \overline{p_{m+1}}, \ldots, p_{s}, \overline{p_{s}}\right\} .
$$

The set of critical points of $f_{\mid W_{\mathbf{C}}}$ is $V_{\mathbf{C}}$ and $V_{\mathbf{R}}=V_{\mathbf{C}} \cap \mathbf{R}^{n}=\left\{p_{1}, \ldots, p_{m}\right\}$ is the set of critical points of $f_{\mid W_{\mathbf{R}}}$. After an appropriate change of coordinates, one may assume that for each $p \in V_{\mathbf{C}}, M(p) \neq 0$. let

Let $\left(x_{1}, \ldots, x_{n} ; \lambda_{1}, \ldots, \lambda_{k} ; \mu\right)$ be a coordinate system in $\mathbf{R}^{n+k+1}$ and

$$
\begin{array}{ccc}
H: \mathbf{R}^{n+k+1} & \rightarrow & \mathbf{R}^{n+k+1} \\
\left(x_{1}, \ldots, x_{n} ; \lambda_{1}, \ldots, \lambda_{k} ; \mu\right) & \mapsto \quad\left(\nabla f+\sum_{i=1}^{k} \lambda_{i} \nabla F_{i}+\mu \nabla g, F_{1}, \ldots, F_{k}, g\right) .
\end{array}
$$


Let $B_{\mathbf{R}}=\frac{\mathbf{R}[x ; \lambda ; \mu]}{(H)}$ and assume that $\operatorname{dim}_{\mathbf{R}} B_{\mathbf{R}}<+\infty$. Let $Y_{\mathbf{R}}=$ $\left\{(q ; \lambda ; \mu) \in \mathbf{R}^{n+k+1} / H(q, \lambda, \mu)=0\right\}$. Then $Y_{\mathbf{R}}$ is a finite set, say

$$
Y_{\mathbf{R}}=\left\{\left(q_{1}, \lambda_{1}, \mu_{1}\right), \ldots,\left(q_{l}, \lambda_{l}, \mu_{l}\right)\right\} .
$$

The points $q_{1}, \ldots, q_{l}$ are exactly the critical points of $f_{\mid W_{\mathbf{R}} \cap g^{-1}(0)}$. Now it is clear that Lemma 4.1, Proposition 4.2 and Proposition 4.3 are still true if we replace $\omega$ by $f$.

Let $\phi$ be the global residue on $A_{\mathbf{R}}$ and consider the following bilinear symmetric forms on $A_{\mathbf{R}}$ :

$$
\begin{array}{ccccc}
\Phi & : & A_{\mathbf{R}} \times A_{\mathbf{R}} \rightarrow \mathbf{R} & \text { defined by } & \Phi\left(g_{1}, g_{2}\right)=\phi\left(g_{1} g_{2}\right), \\
\Phi_{g} & : & A_{\mathbf{R}} \times A_{\mathbf{R}} \rightarrow \mathbf{R} & \text { defined by } & \Phi\left(g_{1}, g_{2}\right)=\phi\left(g g_{1} g_{2}\right), \\
\Phi_{f} & : & A_{\mathbf{R}} \times A_{\mathbf{R}} \rightarrow \mathbf{R} & \text { defined by } & \Phi\left(g_{1}, g_{2}\right)=\phi\left(f g_{1} g_{2}\right), \\
\Phi_{f g} & : & A_{\mathbf{R}} \times A_{\mathbf{R}} \rightarrow \mathbf{R} & \text { defined by } & \Phi\left(g_{1}, g_{2}\right)=\phi\left(g f g_{1} g_{2}\right) .
\end{array}
$$

In the same way, we can define $\Phi^{M}, \Phi_{g}^{M}, \Phi_{f}^{M}$ and $\Phi_{g f}^{M}$.

Let $\Psi$ be the global residue on $B_{\mathbf{R}}$ and consider the following symmetric forms on $B_{\mathbf{R}}$ :

$$
\begin{array}{ccccc}
\Psi & : & B_{\mathbf{R}} \times B_{\mathbf{R}} \rightarrow \mathbf{R} & \text { defined by } & \Psi\left(g_{1}, g_{2}\right)=\psi\left(g_{1} g_{2}\right), \\
\Psi_{f} & : & B_{\mathbf{R}} \times B_{\mathbf{R}} \rightarrow \mathbf{R} & \text { defined by } & \Psi\left(g_{1}, g_{2}\right)=\psi\left(f g_{1} g_{2}\right), \\
\Psi_{\mu} & : & B_{\mathbf{R}} \times B_{\mathbf{R}} \rightarrow \mathbf{R} & \text { defined by } & \Psi\left(g_{1}, g_{2}\right)=\psi\left(\mu g_{1} g_{2}\right), \\
\Psi_{f \mu} & : & B_{\mathbf{R}} \times B_{\mathbf{R}} \rightarrow \mathbf{R} & \text { defined by } & \Psi\left(g_{1}, g_{2}\right)=\psi\left(f \mu g_{1} g_{2}\right) .
\end{array}
$$

Theorem 5.1. Assume that

- $W_{\mathbf{C}}$ is a smooth complex manifold of dimension $n-k$ and $W_{\mathbf{R}}$ is not empty,

- $W_{\mathbf{C}} \cap g_{\mathbf{C}}^{-1}(0)$ is a smooth complex manifold of dimension $n-k-1$ and $W_{\mathbf{R}} \cap g^{-1}(0)$ is not empty,

- for each $p \in V_{\mathbf{C}}, M(p) \neq 0$,

- $\Phi_{g f}$ is non-degenerate,

- $\Psi_{f}$ is non-degenerate,

- $f_{\mid W_{\mathbf{R}}}$ is proper, 
then

1. $W_{\mathbf{R}}$ is a smooth real manifold of dimension $n-k$,

2. $W_{\mathbf{R}} \cap g^{-1}(0)$ is a smooth real manifold of dimension $n-k-1$,

3. $\Phi_{g}, \Phi_{f}, \Phi^{M}, \Phi_{g}^{M}, \Phi_{f}^{M}$ and $\Phi_{f g}^{M}$ are non-degenerate,

4. $W_{\mathbf{R}} \cap f^{-1}(0)$ is either a smooth real manifold of dimension $n-k-1$ or empty,

5. all critical points of $f_{\mid W_{\mathbf{R}}(g \geq 0)}$ and of $f_{\mid W_{\mathbf{R}}(g \leq 0)}$ lying in $W_{\mathbf{R}} \cap$ $g^{-1}(0)$ are correct,

6. $\Psi_{\mu}$ and $\Psi_{\mu f}$ are non-degenerate,

7. $W_{\mathbf{R}} \cap f^{-1}(0) \cap g^{-1}(0)$ is either a smooth real manifold of dimension $n-k-2$ or empty,

8. if $n-k$ is odd

$$
\begin{gathered}
{\left[\begin{array}{cccc}
1 & 1 & 1 & 1 \\
1 & -1 & 1 & -1 \\
1 & 1 & -1 & -1 \\
1 & -1 & -1 & 1
\end{array}\right] \times\left[\begin{array}{l}
\chi_{\geq, \geq} \\
\chi_{\geq, \leq} \\
\chi_{\leq, \geq} \\
\chi_{\leq, \leq}
\end{array}\right]-2 \times\left[\begin{array}{c}
\chi_{\geq,=}+\chi_{\leq,=} \\
0 \\
\chi_{\geq,=}-\chi_{\leq,=} \\
0
\end{array}\right]=} \\
(-1)^{k} \times\left[\begin{array}{c}
\text { signature } \Phi_{f}-\text { signature } \Psi \\
\text { signature } \Phi-\text { signature } \Psi_{f} \\
\text { signature } \Phi_{f g}+\text { signature } \Psi_{f \mu} \\
\text { signature } \Phi_{g}+\text { signature } \Psi_{\mu}
\end{array}\right]
\end{gathered}
$$

if $n-k$ is even

$$
\begin{gathered}
{\left[\begin{array}{cccc}
1 & 1 & 1 & 1 \\
1 & -1 & 1 & -1 \\
1 & 1 & -1 & -1 \\
1 & -1 & -1 & 1
\end{array}\right] \times\left[\begin{array}{l}
\chi \geq, \geq \\
\chi \geq, \leq \\
\chi \leq, \geq \\
\chi \leq, \leq
\end{array}\right]-2 \times\left[\begin{array}{c}
\chi_{\geq,=}+\chi_{\leq,=} \\
0 \\
\chi_{\geq,=}-\chi_{\leq,=} \\
0
\end{array}\right]=} \\
{\left[\begin{array}{c}
\text { signature } \Phi^{M}+(-1)^{k+1} \text { signature } \Psi_{f} \\
\text { signature } \Phi_{f}^{M}+(-1)^{k+1} \text { signature } \Psi \\
\text { signature } \Phi_{g}^{M}+(-1)^{k} \text { signature } \Psi_{\mu} \\
\text { signature } \Phi_{f g}^{M}+(-1)^{k} \text { signature } \Psi_{f \mu}
\end{array}\right] .}
\end{gathered}
$$


Proof. 1., 2. and 3. are clear.

Because $\Phi_{g f}$ is non-degenerate then for all $p \in V_{\mathbf{C}}, f(p) \neq 0$ and $W_{\mathbf{R}} \cap f^{-1}(0)$ is smooth or empty which shows 4 . Because $\Phi_{g f}$ is nondegenerate then for all $p \in V_{\mathbf{C}}, g(p) \neq 0$ and this proves $\mathbf{5}$. and this also implies as in Theorem 4.4 that $\Psi_{\mu}$ is non-degenerate. Furthermore if $\Psi_{f}$ is non-degenerate then $\Psi_{\mu f}$ is also non-degenerate and $\mathbf{6}$. is proved. If $\Psi_{f}$ is non-degenerate then for all $(q, \lambda, \mu) \in \mathbf{C}^{n+k+1}$ such that $H(q, \lambda, \mu)=0, f(q) \neq 0$ which implies that $W_{\mathbf{C}} \cap f_{\mathbf{C}}^{-1}(0) \cap g_{\mathbf{C}}^{-1}(0)$ is smooth i.e 7. is shown.

To prove 8., we choose a function $\tilde{f}: W_{\mathbf{R}} \rightarrow \mathbf{R}$ which approximates $f_{\mid W_{\mathbf{R}}}$ in the $C^{2}$-topology such that $\tilde{f}_{\mid W_{\mathbf{R}}(g \geq 0)}$ and $\tilde{f}_{\mid W_{\mathbf{R}}(g \leq 0)}$ are Morse correct functions. For all $j \in\{1, \ldots, n\}$, let $\left\{p_{j 1}, \ldots, p_{j \sigma(j)}\right\}$ be the set of critical points of $\tilde{f}_{\mid W_{\mathbf{R}}}$ lying near $p_{j}$ and let $\left\{\lambda_{j 1}, \ldots, \lambda_{j \sigma(j)}\right\}$ be the set of their respective indices. For all $s \in\{1, \ldots, l\}$, let $\left\{q_{s 1}, \ldots, q_{s \tau(s)}\right\}$ be the set of critical points of $\tilde{f}_{\mid W_{\mathbf{R}} \cap g^{-1}(0)}$ lying near $q_{s}$ and let $\left\{\mu_{s 1}, \ldots, \mu_{s \tau(s)}\right\}$ be the set of their respective indices. Applying Theorem 2.5, we get

$$
\begin{gathered}
\chi_{\geq, \geq}-\chi_{\geq,=}=\sum_{\substack{j / g\left(p_{j}\right)>0 \\
f\left(p_{j}\right)>0}} \sum_{i=1}^{\sigma(j)}(-1)^{\lambda_{j i}}+\sum_{\substack{s / \mu_{s}<0 \\
f\left(q_{s}\right)>0}} \sum_{i=1}^{\tau(s)}(-1)^{\mu_{s i}} \\
\chi_{\geq, \leq}-\chi_{\geq,=}=(-1)^{n-k} \sum_{\substack{j / g\left(p_{j}\right)>0 \\
f\left(p_{j}\right)<0}} \sum_{i=1}^{\sigma(j)}(-1)^{\lambda_{j i}}+(-1)^{n-k-1} \sum_{\substack{s / \mu_{s}>0 \\
f\left(q_{s}\right)<0}} \sum_{i=1}^{\tau(s)}(-1)^{\mu_{s i}} \\
\chi_{\leq, \geq}-\chi_{\leq,=}=\sum_{\substack{j / g\left(p_{j}\right)<0 \\
f\left(p_{j}\right)>0}} \sum_{i=1}^{\sigma(j)}(-1)^{\lambda_{j i}}+\sum_{\substack{s / \mu_{s}>0 \\
f\left(q_{s}\right)>0}} \sum_{i=1}^{\tau(s)}(-1)^{\mu_{s i}}(3) \\
\chi_{\leq, \leq-} \chi_{\leq,=}=(-1)^{n-k} \sum_{\substack{j / g\left(p_{j}\right)<0 \\
f\left(p_{j}\right)<0}} \sum_{i=1}^{\sigma(j)}(-1)^{\lambda_{j i}}+(-1)^{n-k-1} \sum_{\substack{s / \mu_{s}<0 \\
f\left(q_{s}\right)<0}} \sum_{i=1}^{\tau(s)}(-1)^{\mu_{s i}}
\end{gathered}
$$

We prove the case $n-k$ odd. The combination $(1)+(2)+(3)+(4)$ gives

$$
\chi_{\geq, \geq}+\chi_{\geq, \leq}-2 \chi_{\geq,=}+\chi_{\leq, \geq}+\chi_{\leq, \leq}-2 \chi_{\leq,=}=
$$




$$
\sum_{j} \operatorname{sign} f\left(p_{j}\right) \sum_{i=1}^{\sigma(j)}(-1)^{\lambda_{j i}}+\sum_{s} \sum_{i=1}^{\tau(s)}(-1)^{\mu_{s i}} .
$$

Proposition 4.2 and Proposition 4.3 imply

$$
\begin{gathered}
\chi_{\geq, \geq}+\chi_{\geq, \leq}-2 \chi_{\geq,=}+\chi_{\leq, \geq}+\chi_{\leq, \leq}-2 \chi_{\leq,=}= \\
(-1)^{k} \text { signature } \Phi_{f}+(-1)^{k+1} \text { signature } \Psi .
\end{gathered}
$$

In the same way, $(1)-(2)+(3)-(4)$ gives

$$
\begin{gathered}
\chi_{\geq, \geq}-\chi_{\geq, \leq}+\chi_{\leq, \geq}-\chi_{\leq, \leq}=\sum_{j} \sum_{i=1}^{\sigma(j)}(-1)^{\lambda_{j i}}+\sum_{s} \operatorname{sign} f\left(q_{s}\right) \sum_{i=1}^{\tau(s)}(-1)^{\mu_{s i}}= \\
(-1)^{k} \text { signature } \Phi+(-1)^{k+1} \text { signature } \Psi_{f} .
\end{gathered}
$$

Then $(1)+(2)-(3)-(4)$ gives

$$
\begin{gathered}
\chi_{\geq, \geq}+\chi_{\geq, \leq}-2 \chi_{\geq,=}-\chi_{\leq, \geq}-\chi_{\leq, \leq}+2 \chi_{\leq,=}= \\
\sum_{j} \operatorname{sign}(f g)\left(p_{j}\right) \sum_{i=1}^{\sigma(j)}(-1)^{\lambda_{j i}}-\sum_{s} \operatorname{sign}\left(\mu_{s} f\left(q_{s}\right)\right) \sum_{i=1}^{\tau(s)}(-1)^{\mu_{s i}}= \\
(-1)^{k} \text { signature } \Phi_{f g}+(-1)^{k} \text { signature } \Psi_{f \mu} .
\end{gathered}
$$

Finally $(1)-(2)-(3)+(4)$ gives

$$
\begin{gathered}
\chi_{\geq, \geq}-\chi_{\geq, \leq}-\chi_{\leq, \geq}+\chi_{\leq, \leq}=\sum_{j} \operatorname{sign} g\left(p_{j}\right) \sum_{i=1}^{\sigma(j)}(-1)^{\lambda_{j i}}- \\
\sum_{s} \operatorname{sign} \mu_{s} \sum_{i=1}^{\tau(s)}(-1)^{\mu_{s i}}=(-1)^{k} \text { signature } \Phi_{g}+(-1)^{k} \text { signature } \Psi_{\mu} .
\end{gathered}
$$

We prove the case $n-k$ even in the same way.

Now consider the following algebra

$$
C_{\mathbf{R}}=\frac{\mathbf{R}\left[x_{1}, \ldots, x_{n}\right]}{\left(F_{1}, \ldots, F_{k}, f, \frac{\partial\left(g, F_{1}, \ldots, F_{k}, f\right)}{\partial\left(x_{i_{1}}, \ldots, x_{i} x_{k+2}\right)}\right)} .
$$


Assume that $\operatorname{dim}_{\mathbf{R}} C_{\mathbf{R}}<+\infty$ then, using [Dut1] Theorem 2.6 and Corollary 2.7 , it is possible to express $\chi_{\geq,=}-\chi_{\leq,=}$and $\chi_{\geq,=}+\chi_{\leq,=}$in terms of signatures of appropriate bilinear symmetric forms defined on $C_{\mathbf{R}}$.

Remark 5.2. Under finite dimensional conditions and non-degeneracy conditions, it is possible to express $\chi_{*, ?}, *, ? \in\{\leq, \geq\}$, in terms of signatures of bilinear symmetric forms.

Proof. Use the previous theorem, [Dut1] Theorem 2.6 and Corollary 2.7 and the fact that

$$
\operatorname{det}\left[\begin{array}{cccc}
1 & 1 & 1 & 1 \\
1 & -1 & 1 & -1 \\
1 & 1 & -1 & -1 \\
1 & -1 & -1 & 1
\end{array}\right]=4 \neq 0
$$

\subsection{Examples}

Example 1. Let $W_{\mathbf{R}}=\mathbf{R}^{2}, g=x_{1}-1$ and $f=x_{1}^{2}+x_{2}^{2}-4$. It is clear that $f$ is proper. Computations give

- $\operatorname{dim}_{\mathbf{R}} A_{\mathbf{R}}=1$, signature $\Phi=1$, signature $\Phi_{f}=-1$, signature $\Phi_{g}=-1, \operatorname{rank} \Phi_{f g}=1$ and signature $\Phi_{f g}=1$,

- $\operatorname{dim}_{\mathbf{R}} B_{\mathbf{R}}=1$, signature $\Psi=-1$, signature $\Psi_{\mu}=1$, signature $\Psi_{\mu f}=-1, \operatorname{rank} \Psi_{f}=1$ and signature $\Psi_{f}=1$.

So, by Theorem 5.1,

$$
\begin{gathered}
\chi(g \geq 0, f \geq 0)+\chi(g \geq 0, f \leq 0)-2 \chi(g \geq 0, f=0)+ \\
\chi(g \leq 0, f \geq 0)+\chi(g \leq 0, f \leq 0)-2 \chi(g \leq 0, f=0)=0, \\
\chi(g \geq 0, f \geq 0)-\chi(g \geq 0, f \leq 0)+ \\
\chi(g \leq 0, f \geq 0)-\chi(g \leq 0, f \leq 0)=0, \\
\chi(g \geq 0, f \geq 0)+\chi(g \geq 0, f \leq 0)-2 \chi(g \geq 0, f=0)- \\
\chi(g \leq 0, f \geq 0)-\chi(g \leq 0, f \leq 0)+2 \chi(g \leq 0, f=0)=0,
\end{gathered}
$$




$$
\begin{gathered}
\chi(g \geq 0, f \geq 0)-\chi(g \geq 0, f \leq 0)- \\
\chi(g \leq 0, f \geq 0)+\chi(g \leq 0, f \leq 0)=0 .
\end{gathered}
$$

Example 2. Let $W_{\mathbf{R}}=\mathbf{R}^{3}$, let $g=x_{1}^{3}+x_{2} x_{3}+x_{3}^{2}-1$ and let $f=$ $x_{1}^{2}+x_{2}^{2}+x_{3}^{2}-9$. The computer gives

- $\operatorname{dim}_{\mathbf{R}} A_{\mathbf{R}}=1$, signature $\Phi=1$, signature $\Phi_{f}=-1$, signature $\Phi_{g}=-1, \operatorname{rank} \Phi_{f g}=1$ and signature $\Phi_{f g}=1$,

- $\operatorname{dim}_{\mathbf{R}} B_{\mathbf{R}}=11$, signature $\Psi=-1$, signature $\Psi_{\mu}=1$, signature $\Psi_{\mu f}=-1, \operatorname{rank} \Psi_{f}=11$ and signature $\Psi_{f}=1$.

So, by Theorem 5.1,

$$
\begin{gathered}
\chi(g \geq 0, f \geq 0)+\chi(g \geq 0, f \leq 0)-2 \chi(g \geq 0, f=0)+ \\
\chi(g \leq 0, f \geq 0)+\chi(g \leq 0, f \leq 0)-2 \chi(g \leq 0, f=0)=0, \\
\chi(g \geq 0, f \geq 0)-\chi(g \geq 0, f \leq 0)+ \\
\chi(g \leq 0, f \geq 0)-\chi(g \leq 0, f \leq 0)=0, \\
\chi(g \geq 0, f \geq 0)+\chi(g \geq 0, f \leq 0)-2 \chi(g \geq 0, f=0)- \\
\chi(g \leq 0, f \geq 0)-\chi(g \leq 0, f \leq 0)+2 \chi(g \leq 0, f=0)=0, \\
\chi(g \geq 0, f \geq 0)-\chi(g \geq 0, f \leq 0)- \\
\chi(g \leq 0, f \geq 0)+\chi(g \leq 0, f \leq 0)=0 .
\end{gathered}
$$

\section{Study of the case $\Phi_{f}$ degenerate}

Now we investigate the case when $W_{\mathbf{R}} \cap f^{-1}(0)$ has isolated singularities. We keep the notations of the previous section, we put $d=\operatorname{dim}_{\mathbf{R}} A_{\mathbf{R}}$. We set

$$
\begin{aligned}
& e(d)=d \text { and } o(d)=d+1 \text { if } d \text { is even, } \\
& e(d)=d+1 \text { and } o(d)=d \text { if } d \text { is odd. }
\end{aligned}
$$


We define the following bilinear symmetric forms :

$$
\begin{array}{ccc}
\Phi_{f^{e(d)}}: A_{\mathbf{R}} \times A_{\mathbf{R}} \rightarrow \mathbf{R} & \text { defined by } & \Phi_{f^{e(d)}}\left(g_{1}, g_{2}\right)=\phi\left(f^{e(d)} g_{1} g_{2}\right), \\
\Phi_{f^{e(d)} g}: A_{\mathbf{R}} \times A_{\mathbf{R}} \rightarrow \mathbf{R} & \text { defined by } & \Phi_{f^{e(d)}}\left(g_{1}, g_{2}\right)=\phi\left(f^{e(d)} g g_{1} g_{2}\right), \\
\Phi_{f^{o(d)}}: A_{\mathbf{R}} \times A_{\mathbf{R}} \rightarrow \mathbf{R} & \text { defined by } & \Phi_{f^{o(d)}}\left(g_{1}, g_{2}\right)=\phi\left(f^{o(d)} g_{1} g_{2}\right), \\
\Phi_{f^{o(d)} g}: A_{\mathbf{R}} \times A_{\mathbf{R}} \rightarrow \mathbf{R} & \text { defined by } & \Phi_{f^{o(d)} g}\left(g_{1}, g_{2}\right)=\phi\left(f^{o(d)} g g_{1} g_{2}\right) .
\end{array}
$$

In the same way, we can define $\Phi_{f^{e(d)}}^{M}, \Phi_{f^{e(d)} g}^{M}, \Phi_{f^{o(d)}}^{M}$ and $\Phi_{f^{o(d)} g}^{M}$.

Theorem 6.1. Assume that

- $\operatorname{dim}_{\mathbf{R}} A_{\mathbf{R}}<+\infty$ and $\operatorname{dim}_{\mathbf{R}} B_{\mathbf{R}}<+\infty$,

- $W_{\mathbf{C}}$ is a smooth complex manifold of dimension $n-k$ and $W_{\mathbf{R}}$ is non-empty,

- $W_{\mathbf{C}} \cap g_{\mathbf{C}}^{-1}(0)$ is a smooth manifold of dimension $n-k-1$ and $W_{\mathbf{R}} \cap g^{-1}(0)$ is not empty,

- for each $p \in V_{\mathbf{C}}, M(p) \neq 0$,

- $\Phi_{f}$ is degenerate,

- $\Phi_{g}$ and $\Psi_{f}$ are non-degenerate,

- $f_{\mid W_{\mathbf{R}}}$ is proper,

then

1. $W_{\mathbf{R}}$ is a smooth real manifold of dimension $n-k$,

2. $W_{\mathbf{R}} \cap g^{-1}(0)$ is a smooth real manifold of dimension $n-k-1$,

3. $\Phi_{g}^{M}$ is non-degenerate,

4. $\Phi_{f}^{M}$ and $\Phi_{f g}^{M}$ are degenerate,

5. all critical points of $f_{\mid W_{\mathbf{R}}(g \geq 0)}$ and of $f_{\mid W_{\mathbf{R}}(g \leq 0)}$ lying in $W_{\mathbf{R}} \cap$ $g^{-1}(0)$ are correct,

6. $\Psi_{\mu}$ and $\Psi_{\mu f}$ are non-degenerate,

7. $W_{\mathbf{R}} \cap f^{-1}(0)$ have isolated singularities or is smooth of dimension $n-k-1$ or is empty, 
8. $W_{\mathbf{R}} \cap f^{-1}(0) \cap g^{-1}(0)$ is a smooth real manifold of dimension $n-$ $k-2$ or is empty,

9. if $n-k$ is odd

$$
\begin{gathered}
{\left[\begin{array}{cccc}
1 & 1 & 1 & 1 \\
1 & -1 & 1 & -1 \\
1 & 1 & -1 & -1 \\
1 & -1 & -1 & 1
\end{array}\right] \times\left[\begin{array}{l}
\chi_{\geq, \geq} \\
\chi \geq, \leq \\
\chi_{\leq, \geq} \\
\chi_{\leq, \leq}
\end{array}\right]-2 \times\left[\begin{array}{c}
\chi_{\geq,=}+\chi_{\leq,=} \\
0 \\
\chi_{\geq,=}-\chi_{\leq,=} \\
0
\end{array}\right]=} \\
(-1)^{k} \times\left[\begin{array}{c}
\text { signature } \Phi_{f^{o(d)}}-\operatorname{signature} \Psi \\
\text { signature } \Phi_{f^{e(d)}}-\text { signature } \Psi_{f} \\
\text { signature } \Phi_{f^{o(d)} g}+\text { signature } \Psi_{f \mu} \\
\text { signature } \Phi_{f^{e(d)} g}+\text { signature } \Psi_{\mu}
\end{array}\right],
\end{gathered}
$$

if $n-k$ is even

$$
\begin{gathered}
{\left[\begin{array}{cccc}
1 & 1 & 1 & 1 \\
1 & -1 & 1 & -1 \\
1 & 1 & -1 & -1 \\
1 & -1 & -1 & 1
\end{array}\right] \times\left[\begin{array}{l}
\chi_{\geq, \geq} \\
\chi_{\geq, \leq} \\
\chi_{\leq, \geq} \\
\chi_{\leq, \leq}
\end{array}\right]-2 \times\left[\begin{array}{c}
\chi_{\geq,=}+\chi_{\leq,=} \\
0 \\
\chi_{\geq,=}-\chi_{\leq,=} \\
0
\end{array}\right]=} \\
\\
{\left[\begin{array}{c}
\text { signature } \Phi_{f^{e(d)}}^{M}+(-1)^{k+1} \text { signature } \Psi_{f} \\
\text { signature } \Phi_{f^{o(d)}}^{M}+(-1)^{k+1} \text { signature } \Psi \\
\text { signature } \Phi_{f^{e(d) g}}^{M}+(-1)^{k} \text { signature } \Psi_{\mu} \\
\text { signature } \Phi_{f^{o(d) g}}^{M}+(-1)^{k} \text { signature } \Psi_{f \mu}
\end{array}\right]}
\end{gathered}
$$

Proof. 1., 2., 3., 5., 6. and 8. are clear.

For 4. and 7. use Theorem 3.4.

For 9. we proceed as we did in Theorem 5.1 and we use Proposition 3.6. For example, in order to prove, in the case $n-k$ odd, that

$$
\begin{gathered}
\chi_{\geq, \geq}+\chi_{\geq, \leq}+\chi_{\leq, \geq}+\chi_{\leq, \leq}-2 \chi_{\geq,=}-2 \chi_{\leq,=}= \\
(-1)^{k} \text { signature } \Phi_{f^{o(d)}}-\text { signature } \Psi,
\end{gathered}
$$


we first notice that, keeping the notations introduced in the proof of Theorem 5.1,

$$
\begin{gathered}
\chi_{\geq, \geq}+\chi_{\geq, \leq}-2 \chi_{\geq,=}+\chi_{\leq, \geq}+\chi_{\leq, \leq}-2 \chi_{\leq,=}= \\
\sum_{j / f\left(p_{j}\right) \neq 0} \operatorname{sign} f\left(p_{j}\right) \sum_{i=1}^{\sigma(j)}(-1)^{\lambda_{j i}}+\sum_{s} \sum_{i=1}^{\tau(s)}(-1)^{\mu_{s i}} .
\end{gathered}
$$

Using Proposition 4.2, we have

$$
\begin{gathered}
\sum_{j / f\left(p_{j}\right) \neq 0} \operatorname{sign} f\left(p_{j}\right) \sum_{i=1}^{\sigma(j)}(-1)^{\lambda_{j i}}=\sum_{j / f\left(p_{j}\right) \neq 0} \operatorname{sign} f\left(p_{j}\right)^{o(d)} \sum_{i=1}^{\sigma(j)}(-1)^{\lambda_{j i}}= \\
(-1)^{k} \sum_{j / f\left(p_{j}\right) \neq 0} \text { signature } \Phi_{f^{o(d)}}^{j} .
\end{gathered}
$$

By Proposition 3.6, we obtain

$$
\sum_{j / f\left(p_{j}\right) \neq 0} \operatorname{sign} f\left(p_{j}\right) \sum_{i=1}^{\sigma(j)}(-1)^{\lambda_{j i}}=(-1)^{k} \text { signature } \Phi_{f^{o(d)}} .
$$

By Proposition 4.3, we still have

$$
\sum_{s} \sum_{i=1}^{\tau(s)}(-1)^{\mu_{s i}}=(-1)^{k+1} \text { signature } \Psi .
$$

Now using the results of Section 4, we can express

$$
\begin{aligned}
& \chi\left(W_{\mathbf{R}}(g \geq 0)\right)+\chi\left(W_{\mathbf{R}}(g \leq 0)\right), \\
& \chi\left(W_{\mathbf{R}}(g \geq 0)\right)-\chi\left(W_{\mathbf{R}}(g \leq 0)\right),
\end{aligned}
$$

in terms of signatures of suitable bilinear symmetric forms. We will write 
- if $n-k$ is odd

$$
\begin{gathered}
\chi\left(W_{\mathbf{R}}(g \geq 0)\right)+\chi\left(W_{\mathbf{R}}(g \leq 0)\right)= \\
(-1)^{k}\left(\text { signature } \Phi^{\omega}-\text { signature } \Psi^{\omega}\right), \\
\chi\left(W_{\mathbf{R}}(g \geq 0)\right)-\chi\left(W_{\mathbf{R}}(g \leq 0)\right)= \\
(-1)^{k}\left(\text { signature } \Phi_{g}^{\omega}-\text { signature } \Psi_{\mu}^{\omega}\right),
\end{gathered}
$$

- if $n-k$ is even

$$
\chi\left(W_{\mathbf{R}}(g \geq 0)\right)+\chi\left(W_{\mathbf{R}}(g \leq 0)\right)=
$$

signature $\Phi^{M, \omega}+(-1)^{k+1}$ signature $\Psi^{\omega}$,

$$
\chi\left(W_{\mathbf{R}}(g \geq 0)\right)-\chi\left(W_{\mathbf{R}}(g \leq 0)\right)=
$$

signature $\Phi_{g}^{M, \omega}+(-1)^{k}$ signature $\Psi_{\mu}^{\omega}$,

where these bilinear symmetrics forms are defined on

$$
\frac{\mathbf{R}\left[x_{1}, \ldots, x_{n}\right]}{\left(F_{1}, \ldots, F_{k}, \frac{\partial\left(\omega, F_{1}, \ldots, F_{k}\right)}{\partial\left(x_{i_{1}}, \ldots, x_{i_{k+1}}\right)}\right)} \text { or } \frac{\mathbf{R}\left[x_{1}, \ldots, x_{n} ; \lambda_{1}, \ldots, \lambda_{k}, \mu\right]}{\left(\nabla \omega+\sum_{i=1}^{k} \lambda_{i} \nabla F_{i}+\mu \nabla g, F_{1}, \ldots, F_{k}, g\right)} \text {. }
$$

Now we are able to give a formula for a semi-algebraic set which is the intersection of a compact algebraic complete intersection with isolated singularities and a polynomial inequality.

Corollary 6.2. Under the assumptions of Theorem 4.4 and Theorem 6.1, we can express $\chi_{\geq,=}$and $\chi_{\leq,=}$in terms of signatures. If $n-k$ is odd

$(-1)^{k}\left(\right.$ signature $\Phi^{\omega}-$ signature $\Psi^{\omega}-$ signature $\Phi_{f^{o(d)}}+$ signature $\left.\Psi\right)=$

$$
\chi_{\geq,=}+\chi_{\leq,=},
$$

and

$(-1)^{k}\left(\right.$ signature $\Phi_{g}^{\omega}+$ signature $\Psi_{\mu}^{\omega}$-signature $\Phi_{f^{o(d)} g}$-signature $\left.\Psi_{f \mu}\right)=$ $\chi_{\geq,=}=-\chi_{\leq,=}$. 
If $n-k$ is even

$$
\begin{gathered}
\text { signature } \Phi^{M, \omega}+(-1)^{k+1} \text { signature } \Psi^{\omega}-\text { signature } \Phi_{f(d)}^{M}- \\
(-1)^{k+1} \text { signature } \Psi_{f}=\chi_{\geq,=}+\chi_{\leq,=},
\end{gathered}
$$

and

$$
\begin{gathered}
\text { signature } \Phi_{g}^{M, \omega}+(-1)^{k} \text { signature } \Psi_{\mu}^{\omega}-\text { signature } \Phi_{f^{e(d)} g}^{M}- \\
(-1)^{k} \text { signature } \Psi_{\mu}=\chi_{\geq,=}-\chi_{\leq,=} .
\end{gathered}
$$

Proof. Suppose $n-k$ is odd. By Mayer-Vietoris sequence, we have

$$
\begin{aligned}
& \chi\left(W_{\mathbf{R}}(g \geq 0)\right)=\chi_{\geq, \geq}+\chi_{\geq, \leq}-\chi_{\geq,=}, \\
& \chi\left(W_{\mathbf{R}}(g \leq 0)\right)=\chi_{\leq, \geq}+\chi_{\leq, \leq}-\chi_{\leq,=} .
\end{aligned}
$$

So

$$
\begin{gathered}
\chi\left(W_{\mathbf{R}}(g \geq 0)\right)+\chi\left(W_{\mathbf{R}}(g \leq 0)\right)=\chi_{\geq, \geq}+\chi_{\geq, \leq}- \\
\chi_{\geq,=}+\chi_{\leq, \geq}+\chi_{\leq, \leq}-\chi_{\leq,=} .
\end{gathered}
$$

Combining with the first equality in Theorem 6.1 , we obtain

$$
\begin{gathered}
\chi\left(W_{\mathbf{R}}(g \geq 0)\right)+\chi\left(W_{\mathbf{R}}(g \leq 0)\right)-(-1)^{k}\left(\text { signature } \Phi_{f o(d)}-\text { signature } \Psi\right)= \\
\chi_{\geq,=}+\chi_{\leq,=} .
\end{gathered}
$$

Using Theorem 4.4, we get

$$
\chi_{\geq,=}+\chi_{\leq,=}=
$$

$(-1)^{k}\left(\right.$ signature $\Phi^{\omega}-$ signature $\Psi^{\omega}-$ signature $\Phi_{f^{o(d)}}+$ signature $\left.\Psi\right)$.

Now if we express $\chi_{\geq}=-\chi_{\leq,=}$, we obtain the second relation. 


\subsection{Example}

Let $W_{\mathbf{R}}=\mathbf{R}^{2}$, let $g=x_{1}^{3}+x_{2}+1$ and let $f=\left(x_{1}^{2}+x_{2}^{2}-4\right) \times\left(\left(x_{1}-\right.\right.$ $\left.2)^{2}+x_{2}^{2}-9\right)$. Since $|f(x)| \rightarrow+\infty$ as $\|x\| \rightarrow+\infty, f$ is proper. Now Lecki's program gives

- $\operatorname{dim}_{\mathbf{R}} A_{\mathbf{R}}=5, \operatorname{rank} \Phi_{f}=3$ so $\Phi_{f}$ is degenerate, rank $\Phi_{g}=5$ so $\Phi_{g}$ is non-degenerate,

- signature $\Phi_{f^{5}}=-1$, signature $\Phi_{f^{6}}=3$, signature $\Phi_{f^{5} g}=1$ and signature $\Phi_{f^{6} g}=1$,

- $\operatorname{dim}_{\mathbf{R}} B_{\mathbf{R}}=11$, rank $\Psi_{f}=11$, signature $\Psi_{f}=3$, signature $\Psi=$ -1 , signature $\Psi_{\mu}=-1$ and signature $\Psi_{\mu f}=-1$.

When we apply Theorem 6.1, we obtain

$$
\begin{gathered}
\chi(g \geq 0, f \geq 0)+\chi(g \geq 0, f \leq 0)-2 \chi(g \geq 0, f=0)+ \\
\chi(g \leq 0, f \geq 0)+\chi(g \leq 0, f \leq 0)-2 \chi(g \leq 0, f=0)=-4, \\
\chi(g \geq 0, f \geq 0)-\chi(g \geq 0, f \leq 0)+ \\
\chi(g \leq 0, f \geq 0)-\chi(g \leq 0, f \leq 0)=4, \\
\chi(g \geq 0, f \geq 0)+\chi(g \geq 0, f \leq 0)-2 \chi(g \geq 0, f=0)- \\
\chi(g \leq 0, f \geq 0)-\chi(g \leq 0, f \leq 0)+2 \chi(g \leq 0, f=0)=0, \\
\chi(g \geq 0, f \geq 0)-\chi(g \geq 0, f \leq 0)- \\
\chi(g \leq 0, f \geq 0)+\chi(g \leq 0, f \leq 0)=0 .
\end{gathered}
$$

\section{The case of surfaces}

In this section, we study the case of semi-algebraic sets defined as an intersection of a smooth algebraic surface with two polynomial inequalities. Let $F=\left(F_{1}, \ldots, F_{n-2}\right): \mathbf{R}^{n} \rightarrow \mathbf{R}^{n-2}$ be a polynomial mapping such that $W_{\mathbf{C}}=F_{\mathbf{C}}^{-1}(0)$ is a smooth complex manifold of dimension 2 . Let $W_{\mathbf{R}}=F^{-1}(0)$. Let

$$
M=\frac{\partial\left(F_{1}, \ldots, F_{n-2}\right)}{\partial\left(x_{1}, \ldots, x_{n-2}\right)} .
$$


Let $g_{1}, g_{2}: \mathbf{R}^{n} \rightarrow \mathbf{R}$ be two polynomials and set $g=g_{1} \times g_{2}$. Let $I \subset \mathbf{R}[x]$ be the ideal generated by $F_{1}, \ldots, F_{n-2}$ and all $(n-1) \times(n-1)$ minors

$$
\frac{\partial\left(g, F_{1}, \ldots, F_{n-2}\right)}{\partial\left(x_{i_{1}}, \ldots, x_{i_{n-1}}\right)} \text {. }
$$

Let $A_{\mathbf{R}}=\frac{\mathbf{R}[x]}{I}$. Assume that $\operatorname{dim}_{\mathbf{R}} A_{\mathbf{R}}<+\infty$. We will prove at the end of the section that this condition is generic. We put $d=\operatorname{dim}_{\mathbf{R}} A_{\mathbf{R}}$. Let $V_{\mathbf{C}}=\left\{p \in \mathbf{C}^{n} /\right.$ for all $\left.u \in I u(p)=0\right\}$. It is a finite set and we can write

$$
V_{\mathbf{C}}=\left\{p_{1}, \ldots, p_{m}\right\} \cup\left\{p_{m+1}, \overline{p_{m+1}}, \ldots, p_{s}, \overline{p_{s}}\right\} .
$$

The set of critical points of $g_{\mid W_{\mathbf{R}}}$ is

$$
V_{\mathbf{R}}=V_{\mathbf{C}} \cap \mathbf{R}^{n}=\left\{p_{1}, \ldots, p_{m}\right\} .
$$

After an appropriate change of coordinates, one may assume that for each $p \in V_{\mathbf{C}}, M(p) \neq 0$.

Let $\phi: A_{\mathbf{R}} \rightarrow \mathbf{R}$ be the global residue on $A_{\mathbf{R}}$ and we define the following bilinear symmetric forms :

$$
\begin{array}{ccc}
\Phi_{g^{e(d)}}^{M}: A_{\mathbf{R}} \times A_{\mathbf{R}} \rightarrow \mathbf{R} & \text { defined by } & \Phi_{g^{(e d)}}^{M}\left(l_{1}, l_{2}\right)=\phi\left(M g^{e(d)} l_{1} l_{2}\right), \\
\Phi_{g^{o(d)}}^{M}: A_{\mathbf{R}} \times A_{\mathbf{R}} \rightarrow \mathbf{R} & \text { defined by } & \Phi_{g^{o(d)}}^{M}\left(l_{1}, l_{2}\right)=\phi\left(M g^{o(d)} l_{1} l_{2}\right), \\
\Phi_{g_{1} g^{e(d)}}^{M}: A_{\mathbf{R}} \times A_{\mathbf{R}} \rightarrow \mathbf{R} & \text { defined by } & \Phi_{g_{1} g^{e(d)}}^{M}\left(l_{1}, l_{2}\right)=\phi\left(M g_{1} g^{e(d)} l_{1} l_{2}\right), \\
\Phi_{g_{2} g^{e(d)}}^{M}: A_{\mathbf{R}} \times A_{\mathbf{R}} \rightarrow \mathbf{R} & \text { defined by } & \Phi_{g_{2} g^{e(d)}}^{M}\left(l_{1}, l_{2}\right)=\phi\left(M g_{2} g^{e(d)} l_{1} l_{2}\right), \\
\Phi_{g_{1} g^{o(d)}}^{M}: A_{\mathbf{R}} \times A_{\mathbf{R}} \rightarrow \mathbf{R} & \text { defined by } & \Phi_{g_{1} g^{o(d)}}^{M}\left(l_{1}, l_{2}\right)=\phi\left(M g_{1} g^{o(d)} l_{1} l_{2}\right), \\
\Phi_{g_{2} g^{o(d)}}^{M}: A_{\mathbf{R}} \times A_{\mathbf{R}} \rightarrow \mathbf{R} & \text { defined by } & \Phi_{g_{2} g^{o(d)}}^{M}\left(l_{1}, l_{2}\right)=\phi\left(M g_{2} g^{o(d)} l_{1} l_{2}\right),
\end{array}
$$

We will denote $\chi\left(W_{\mathbf{R}} \cap\left\{g_{1} * 0\right\} \cap\left\{g_{2} ? 0\right\}\right)$ by $\chi_{*, ?}$ where $*, ? \in\{\geq, \leq,=\}$.

Theorem 7.1. Assume that

- $W_{\mathbf{C}}$ is a smooth complex manifold of dimension 2 and $W_{\mathbf{R}}$ is not empty,

- $W_{\mathbf{C}} \cap g_{1}^{-1}(0)$ is a smooth complex manifold of dimension 1 and $W_{\mathbf{R}} \cap g_{1}^{-1}(0)$ is not empty, 
- $W_{\mathbf{C}} \cap g_{2}^{-1}(0)$ is a smooth complex manifold of dimension 1 and $W_{\mathbf{R}} \cap g_{2}^{-1}(0)$ is not empty,

- $W_{\mathbf{C}} \cap g_{1}^{-1}(0)$ and $W_{\mathbf{C}} \cap g_{2}^{-1}(0)$ intersect transversally,

- $g_{\mid W_{\mathbf{R}}}$ is proper,

then

1. $W_{\mathbf{R}}$ is a smooth surface,

2.

$$
\begin{gathered}
{\left[\begin{array}{cccc}
1 & 1 & 1 & 1 \\
1 & -1 & 1 & -1 \\
1 & 1 & -1 & -1 \\
1 & -1 & -1 & 1
\end{array}\right] \times\left[\begin{array}{l}
\chi \geq, \geq \\
\chi_{\geq, \leq} \\
\chi_{\leq, \geq} \\
\chi_{\leq, \leq}
\end{array}\right]-2 \times\left[\begin{array}{c}
\chi_{\geq,=}+\chi_{\leq,=} \\
0 \\
\chi_{\geq,=}-\chi_{\leq,=} \\
0
\end{array}\right]-} \\
2 \times\left[\begin{array}{c}
\chi_{=, \geq}+\chi_{=, \leq} \\
\chi_{=, \geq}-\chi_{=, \leq} \\
0 \\
0
\end{array}\right]+4 \times\left[\begin{array}{c}
\chi_{=,=} \\
0 \\
0 \\
0
\end{array}\right]=\left[\begin{array}{c}
\text { signature } \Phi_{g^{e(d)}}^{M} \\
\text { signature } \Phi_{g_{2} g^{e(d)}}^{M} \\
\text { signature } \Phi_{g_{1} g^{e(d)}}^{M} \\
\text { signature } \Phi_{g^{o(d)}}^{M}
\end{array}\right]
\end{gathered}
$$

Proof. 1. is clear.

Consider the set $W_{\mathbf{R}} \cap\left\{g_{1} \geq 0, g_{2} \geq 0\right\}$. The function $g=g_{1} g_{2}$ is a carpeting function for this manifold with corners, this means that there is a homotopy equivalence between

$$
\left(W_{\mathbf{R}} \cap\left\{g_{1} \geq 0, g_{2} \geq 0\right\}, W_{\mathbf{R}} \cap\left(\left\{g_{1} \geq 0, g_{2}=0\right\} \cup\left\{g_{1}=0, g_{2} \geq 0\right\}\right)\right),
$$

and

$$
\left(W_{\mathbf{R}}(g \geq \varepsilon) \cap\left\{g_{1} \geq 0, g_{2} \geq 0\right\}, W_{\mathbf{R}}(g=\varepsilon) \cap\left\{g_{1} \geq 0, g_{2} \geq 0\right\}\right),
$$

for $\varepsilon>0$ sufficiently small. We thus have

$\chi\left(W_{\mathbf{R}} \cap\left\{g_{1} \geq 0, g_{2} \geq 0\right\}, W_{\mathbf{R}} \cap\left(\left\{g_{1} \geq 0, g_{2}=0\right\} \cup\left\{g_{1}=0, g_{2} \geq 0\right\}\right)\right)=$ 


$$
\begin{gathered}
\chi\left(W_{\mathbf{R}}(g \geq \varepsilon) \cap\left\{g_{1} \geq 0, g_{2} \geq 0\right\}, W_{\mathbf{R}}(g=\varepsilon) \cap\left\{g_{1} \geq 0, g_{2} \geq 0\right\}\right)= \\
\sum_{\substack{j / g_{1}\left(p_{j}\right)>0 \\
g_{2}\left(p_{j}\right)>0}} \sum_{i=1}^{\sigma(j)}(-1)^{\lambda_{j i}}
\end{gathered}
$$

where $\left\{\lambda_{j i}\right\}$ is the set of indices of the non-degenerate critical points $\left\{p_{j i}\right\}$ lying near $p_{j}$ of a Morse approximation $\tilde{g}$ of $g_{\mid W_{\mathbf{R}}}$. In the same way, we have :

$$
\begin{gathered}
\chi\left(W_{\mathbf{R}} \cap\left\{g_{1} \leq 0, g_{2} \leq 0\right\}, W_{\mathbf{R}} \cap\left(\left\{g_{1} \leq 0, g_{2}=0\right\} \cup\left\{g_{1}=0, g_{2} \leq 0\right\}\right)\right)= \\
\chi\left(W_{\mathbf{R}}(g \geq \varepsilon) \cap\left\{g_{1} \leq 0, g_{2} \leq 0\right\}, W_{\mathbf{R}}(g=\varepsilon) \cap\left\{g_{1} \leq 0, g_{2} \leq 0\right\}\right) \\
\sum_{\substack{j / g_{1}\left(p_{j}\right)<0 \\
g_{2}\left(p_{j}\right)<0}} \sum_{i=1}^{\sigma(j)}(-1)^{\lambda_{j i}} \\
\chi\left(W_{\mathbf{R}} \cap\left\{g_{1} \geq 0, g_{2} \leq 0\right\}, W_{\mathbf{R}} \cap\left(\left\{g_{1} \geq 0, g_{2}=0\right\} \cup\left\{g_{1}=0, g_{2} \leq 0\right\}\right)\right)= \\
\chi\left(W_{\mathbf{R}}(g \leq-\varepsilon) \cap\left\{g_{1} \geq 0, g_{2} \leq 0\right\}, W_{\mathbf{R}}(g=-\varepsilon) \cap\left\{g_{1} \geq 0, g_{2} \leq 0\right\}\right) \\
\sum_{\substack{j / g_{1}\left(p_{j}\right)>0 \\
g_{2}\left(p_{j}\right)<0}} \sum_{i=1}^{\sigma(j)}(-1)^{\lambda_{j i}} \\
\chi\left(W_{\mathbf{R}} \cap\left\{g_{1} \leq 0, g_{2} \geq 0\right\}, W_{\mathbf{R}} \cap\left(\left\{g_{1} \leq 0, g_{2}=0\right\} \cup\left\{g_{1}=0, g_{2} \geq 0\right\}\right)\right)= \\
\chi\left(W_{\mathbf{R}}(g \leq-\varepsilon) \cap\left\{g_{1} \leq 0, g_{2} \geq 0\right\}, W_{\mathbf{R}}(g=-\varepsilon) \cap\left\{g_{1} \leq 0, g_{2} \geq 0\right\}\right) \\
\sum_{\substack{j / g_{1}\left(p_{j}\right)<0 \\
g_{2}\left(p_{j}\right)>0}} \sum_{i=1}^{\sigma(j)}(-1)^{\lambda_{j i}}
\end{gathered}
$$

Now the combinations $(1)+(2)+(3)+(4),(1)-(2)+(3)-(4),(1)-$ $(2)-(3)+(4)$ and $(1)+(2)-(3)-(4)$ give the desired formulas.

Using [Dut1] Theorem 2.6, one can express

$$
\chi_{\geq,=} \pm \chi_{\leq,=},
$$


and

$$
\chi=, \geq \pm \chi=, \leq,
$$

in terms of signatures of appropriate bilinear symmetric forms. Furthermore, using the generalized Hermite form, one can express

$$
\chi=,==\chi\left(W_{\mathbf{R}}\left(g_{1}=0, g_{2}=0\right)\right)=\sharp W_{\mathbf{R}} \cap g_{1}^{-1}(0) \cap g_{2}^{-1}(0),
$$

as a signature on the algebra $\frac{\mathbf{R}\left[x_{1}, \ldots, x_{n}\right]}{\left(F_{1}, \ldots, F_{n-2}, g_{1}, g_{2}\right)}$ (see [GRRT], [PSR], [Ro]).

Remark 7.2. Under conditions of theorem 7.1 and some other conditions of finitude and non-degeneracy, one can express the Euler characteristics $\chi_{*, \text { ? }}$ in terms of signatures of suitable bilinear symmetric forms.

Proof. Use the previous remarks, the previous theorem and the fact that

$$
\operatorname{det}\left[\begin{array}{cccc}
1 & 1 & 1 & 1 \\
1 & -1 & 1 & -1 \\
1 & 1 & -1 & -1 \\
1 & -1 & -1 & 1
\end{array}\right]=4 \neq 0
$$

\subsection{Genericity of the finitude condition}

In this section, we prove the "genericity" of the condition $\operatorname{dim}_{\mathbf{R}} \mathbf{R}[x] / I<$ $+\infty$ where $I$ is the ideal generated by $F_{1}, \ldots, F_{n-2}$ and all minors

$$
\frac{\partial\left(g_{1} g_{2}, F_{1}, \ldots, F_{n-2}\right)}{\partial\left(x_{i_{1}}, \ldots, x_{i_{n-1}}\right)} .
$$

We will need the following version of Sard's lemma (see $[\mathrm{BCR}],[\mathrm{BR}]$ ).

Lemma 7.3. Let $M \subset \mathbf{R}^{N}$ be a real constructible set and let $M_{\mathbf{C}}$ be its complexification. Assume that $M_{\mathbf{C}}$ is a smooth complex manifold of dimension $k$. Let $\Pi: \mathbf{R}^{n} \rightarrow \mathbf{R}^{k}$ be a polynomial mapping and let $\Pi_{\mathbf{C}}$ be its complexification. Then for almost all $\alpha \in \mathbf{R}^{k}, \Pi_{\mathbf{C}}^{-1}(\alpha) \cap M_{\mathbf{C}}$ is a finite set of points.

Proof. Let $\Sigma_{\mathbf{C}}$ be the critical set of $\Pi_{\mathbf{C} \mid M_{\mathbf{C}}}$. Then $\Pi_{\mathbf{C}}\left(\Sigma_{\mathbf{C}}\right)$ is a constructible set of $\mathbf{C}^{k}$ of complex dimension at most $k-1$ and $\mathbf{R}^{k} \cap \Pi_{\mathbf{C}}\left(\Sigma_{\mathbf{C}}\right)$ is a real constructible set of dimension at most $k-1$, so for $\alpha \in \mathbf{R}^{k} \backslash$ $\Pi_{\mathbf{C}}\left(\Sigma_{\mathbf{C}}\right), \alpha$ is a regular value of $\Pi_{\mathbf{C}}: M_{\mathbf{C}} \rightarrow \mathbf{C}^{k}$. 
In order to prove the genericity of the condition, we first recall that, by Lemma 4.1 , a polynomial $g_{\mid W_{\mathbf{C}}}$ admits a critical point at $p \in W_{\mathbf{C}} \backslash$ $\left\{M_{\mathbf{C}}=0\right\}$ if and only if the minors

$$
\frac{\partial\left(g, F_{1}, \ldots, F_{k}\right)}{\partial\left(x_{1}, \ldots, x_{n-2}, x_{n-1}\right)} \text { and } \frac{\partial\left(g, F_{1}, \ldots, F_{k}\right)}{\partial\left(x_{1}, \ldots, x_{n-2}, x_{n}\right)}
$$

vanish at $p$.

Let $g_{1}, g_{2}: \mathbf{R}^{n} \rightarrow \mathbf{R}$ be two polynomials. Let $\left(x_{1}, \ldots, x_{n} ; t_{n-1}, t_{n} ; u_{n-1}, u_{n}\right)=(x ; t ; u)$ be a coordinate system in $\mathbf{R}^{n+4}$ and let

$$
\begin{aligned}
& G_{1}(x, t, u)=g_{1}+t_{n-1} x_{n-1}+t_{n} x_{n}, \\
& G_{2}(x, t, u)=g_{2}+u_{n-1} x_{n-1}+u_{n} x_{n} .
\end{aligned}
$$

Let us consider the following polynomial map :

$$
\begin{aligned}
& H=\left(H_{1}, H_{2}\right) \quad: \quad \mathbf{R}^{n+4} \quad \rightarrow \quad \mathbf{R}^{n} \\
& (x ; t ; u) \mapsto\left(F, \frac{\partial\left(\left(G_{1} G_{2}\right), F_{1}, \ldots, F_{n-2}\right)}{\partial\left(x_{1}, \ldots, x_{n-2}, x_{n-1}\right)},\right. \\
& \left.\frac{\left(\partial\left(\left(G_{1} G_{2}\right), F_{1}, \ldots, F_{n-2}\right)\right.}{\partial\left(x_{1}, \ldots, x_{n-2}, x_{n}\right)}\right),
\end{aligned}
$$

which we shall write, for convenience, $H=\left(F, \frac{\partial(G, F)}{\partial\left(x^{\prime}, x_{n-1}\right)}, \frac{\partial(G, F)}{\partial\left(x^{\prime}, x_{n-1}\right)}\right)$. We have

$$
\begin{gathered}
H_{1}(x, t, u)=\frac{\partial\left(g_{1} G_{2}, F\right)}{\partial\left(x^{\prime}, x_{n-1}\right)}+t_{n-1} M G_{2}+t_{n-1} x_{n-1} \frac{\partial\left(G_{2}, F\right)}{\partial\left(x^{\prime}, x_{n-1}\right)}+ \\
t_{n} x_{n} \frac{\partial\left(G_{2}, F\right)}{\partial\left(x^{\prime}, x_{n-1}\right)}, \\
H_{2}(x, t, u)=\frac{\partial\left(g_{1} G_{2}, F\right)}{\partial\left(x^{\prime}, x_{n}\right)}+t_{n-1} x_{n-1} \frac{\partial\left(G_{2}, F\right)}{\partial\left(x^{\prime}, x_{n}\right)}+t_{n} M G_{2}+t_{n} x_{n} \frac{\partial\left(G_{2}, F\right)}{\partial\left(x^{\prime}, x_{n}\right)} .
\end{gathered}
$$

The jacobian matrix Jac $(H)$ has the following form

$$
\operatorname{Jac}(H)=\left(\begin{array}{ccccc}
M & 0 & 0 & 0 & 0 \\
* & M G_{2}+x_{n-1} \frac{\partial\left(G_{2}, F\right)}{\partial\left(x^{\prime}, x_{n-1}\right)} & x_{n} \frac{\partial\left(G_{2}, F\right)}{\partial\left(x^{\prime}, x_{n}-1\right)} & * & * \\
* & x_{n-1} \frac{\partial\left(G_{2}, F\right)}{\partial\left(x^{\prime}, x_{n}\right)} & M G_{2}+x_{n} \frac{\partial\left(G_{2}, F\right)}{\partial\left(x^{\prime}, x_{n}\right)} & * & *
\end{array}\right) .
$$

Hence $Y=H^{-1}(0) \backslash\left\{M G_{2}\left(M G_{2}+x_{n-1} \frac{\partial\left(G_{2}, F\right)}{\partial\left(x^{\prime}, x_{n-1}\right)}+x_{n} \frac{\partial\left(G_{2}, F\right)}{\partial\left(x^{\prime}, x_{n}\right)}\right)=0\right\}$ is a smooth manifold of dimension 4 . Let

$$
\Pi: \begin{aligned}
\mathbf{R}^{n+4} & \rightarrow \mathbf{R}^{4} \\
(x ; t ; u) & \mapsto(t ; u) .
\end{aligned}
$$


Using the above lemma, we can choose $\left(\alpha_{n-1}, \alpha_{n}, \beta_{n-1}, \beta_{n}\right) \in \mathbf{R}^{4}$ close to $(0,0,0,0)$ such that $\Pi_{\mathbf{C}}^{-1}\left(\left(\alpha_{n-1}, \alpha_{n}, \beta_{n-1}, \beta_{n}\right)\right) \cap Y_{\mathbf{C}}$ is finite. Call $\tilde{g}_{1}=g_{1}+\alpha_{n-1} x_{n-1}+\alpha_{n} x_{n}$ and $\tilde{g}_{2}=g_{2}+\beta_{n-1} x_{n-1}+\beta_{n} x_{n}$. We have shown that outside the algebraic set $A=\left\{M \tilde{g}_{2}\left(M \tilde{g}_{2}+x_{n-1} \frac{\partial\left(\tilde{g}_{2}, F\right)}{\partial\left(x^{\prime}, x_{n-1}\right)}+\right.\right.$ $\left.\left.x_{n} \frac{\partial\left(\tilde{g}_{2}, F\right)}{\partial\left(x^{\prime}, x_{n}\right)}\right)=0\right\}$, the system $F=0, H_{1}=0, H_{2}=0$ has a finite number of solutions. Since outside $A, M_{\mathbf{C}} \neq 0$, this means that, by the above remark, $\tilde{g_{1}} \times \tilde{g_{2}}$ has a finite number of critical points on $W_{\mathbf{C}}$ outside the set $\left\{M \tilde{g}_{2}\left(M \tilde{g}_{2}+x_{n-1} \frac{\partial\left(\tilde{g}_{2}, F\right)}{\partial\left(x^{\prime}, x_{n-1}\right)}+x_{n} \frac{\partial\left(\tilde{g}_{2}, F\right)}{\partial\left(x^{\prime}, x_{n}\right)}\right)=0\right\}$. The jacobian matrix Jac $(H)$ may also be written

$\operatorname{Jac}(H)=\left(\begin{array}{ccccc}M & 0 & 0 & 0 & 0 \\ * & * & * & M G_{1}+x_{n-1} \frac{\partial\left(G_{1}, F\right)}{\partial\left(x^{\prime}, x_{n-1}\right)} & x_{n} \frac{\partial\left(G_{1}, F\right)}{\partial\left(x^{\prime}, x_{n}-1\right)} \\ * & * & * & x_{n-1} \frac{\partial\left(G_{1}, F\right)}{\partial\left(x^{\prime}, x_{n}\right)} & M G_{1}+x_{n} \frac{\partial\left(G_{1}, F\right)}{\partial\left(x^{\prime}, x_{n}\right)}\end{array}\right)$,

and so, $T=H^{-1}(0) \backslash\left\{M G_{1}\left(M G_{1}+x_{n-1} \frac{\partial\left(G_{1}, F\right)}{\partial\left(x^{\prime}, x_{n-1}\right)}+x_{n} \frac{\partial\left(G_{1}, F\right)}{\partial\left(x^{\prime}, x_{n}\right)}\right)=0\right\}$ is also a smooth manifold of dimension 4 . Repeating the above argument, we can choose $\left(\alpha_{n-1}, \alpha_{n}, \beta_{n-1}, \beta_{n}\right) \in \mathbf{R}^{4}$ such that $\tilde{g_{1}} \times \tilde{g_{2}}$ has a finite number of critical points on $W_{\mathbf{C}}$ outside the set $\left\{M \tilde{g}_{1}\left(M \tilde{g}_{1}+\right.\right.$ $\left.\left.x_{n-1} \frac{\partial\left(\tilde{g}_{1}, F\right)}{\partial\left(x^{\prime}, x_{n-1}\right)}+x_{n} \frac{\partial\left(\tilde{g}_{1}, F\right)}{\partial\left(x^{\prime}, x_{n}\right)}\right)=0\right\}$.

Now we shall prove that for a large choice of $\left(\alpha_{n-1}, \alpha_{n}, \beta_{n-1}, \beta_{n}\right) \in$ $\mathbf{R}^{4}$ the intersection $\{F=0\} \cap\left\{M \tilde{g}_{1}\left(M \tilde{g}_{1}+x_{n-1} \frac{\partial\left(\tilde{g}_{1}, F\right)}{\partial\left(x^{\prime}, x_{n-1}\right)}+x_{n} \frac{\partial\left(\tilde{g}_{1}, F\right)}{\partial\left(x^{\prime}, x_{n}\right)}\right)=\right.$ $0\} \cap\left\{M \tilde{g}_{2}\left(M \tilde{g}_{2}+x_{n-1} \frac{\partial\left(\tilde{g}_{2}, F\right)}{\partial\left(x^{\prime}, x_{n-1}\right)}+x_{n} \frac{\partial\left(\tilde{g}_{2}, F\right)}{\partial\left(x^{\prime}, x_{n}\right)}\right)=0\right\}$ is a finite set outside $\left\{M_{\mathbf{C}}=0\right\}$. We first prove that $\{F=0\} \cap\left\{\tilde{g_{1}}=0\right\} \cap\left\{\tilde{g_{2}}=0\right\}$ is a finite set outside $\left\{M_{\mathbf{C}}=0\right\}$ for almost all $\left(\alpha_{n-1}, \alpha_{n}, \beta_{n-1}, \beta_{n}\right) \in \mathbf{R}^{4}$ close to $(0,0,0,0)$. Consider the following polynomial map

$$
\begin{aligned}
& T: \mathbf{R}^{n+4} \rightarrow \mathbf{R}^{n} \\
& (x, t, u) \mapsto\left(F, G_{1}, G_{2}\right)
\end{aligned}
$$

Its jacobian matrix Jac $(T)$ has the following form

$$
\operatorname{Jac}(T)=\left(\begin{array}{ccccc}
M & 0 & 0 & 0 & 0 \\
* & x_{n-1} & x_{n} & 0 & 0 \\
* & 0 & 0 & x_{n-1} & x_{n}
\end{array}\right)
$$

Hence

$$
Z=T^{-1}(0) \backslash\left\{\{M=0\} \cup\left\{x_{n-1}=0, x_{n}=0\right\}\right\}
$$


is a analytic manifold of dimension 4 and, as we did previously, for almost all $\left(\alpha_{n-1}, \alpha_{n}, \beta_{n-1}, \beta_{n}\right) \in \mathbf{R}^{4},\{F=0\} \cap\left\{\tilde{g_{1}}=0\right\} \cap\left\{\tilde{g_{2}}=0\right\}$ is a finite set outside $\{M=0\} \cup\left\{x_{n-1}=0, x_{n}=0\right\}$. Let $U: \mathbf{R}^{n} \rightarrow \mathbf{R}^{n}$ be defined by $U=\left(F, x_{n-1}, x_{n}\right)$. The jacobian of $U$ is exactly $M$ so if $p \in U^{-1}(0) \cap\{M \neq 0\}, p$ is a simple zero of $U$ so is isolated. This implies that $\{F=0\} \cap\{M \neq 0\} \cap\left\{x_{n-1}=x_{n}=0\right\}$ is finite and so, $\left\{F=\tilde{g_{1}}=\tilde{g_{2}}=0\right\} \cap\{M \neq 0\}$ is also finite.

Now we check that $\{F=0\} \cap\left\{\tilde{g}_{1}=0\right\} \cap\left\{M \tilde{g}_{2}+x_{n-1} \frac{\partial\left(\tilde{g}_{2}, F\right)}{\partial\left(x^{\prime}, x_{n-1}\right)}+\right.$ $\left.x_{n} \frac{\partial\left(\tilde{g}_{2}, F\right)}{\partial\left(x^{\prime}, x_{n}\right)}=0\right\} \quad$ is $\quad$ a finite set outside $\{M=0\}$ for almost all $\left(\alpha_{n-1}, \alpha_{n}, \beta_{n-1}, \beta_{n}\right) \in \mathbf{R}^{4}$. Let

$$
\begin{array}{cccc}
T^{\prime}: \mathbf{R}^{n+4} & \rightarrow & \mathbf{R}^{n} \\
& (x, t, u) & \mapsto & \left(F, G_{1}, M G_{2}+x_{n-1} \frac{\partial\left(G_{2}, F\right)}{\partial\left(x^{\prime}, x_{n-1}\right)}+x_{n} \frac{\partial\left(G_{2}, F\right)}{\partial\left(x^{\prime}, x_{n}\right)}\right)
\end{array},
$$

and let Jac $\left(T^{\prime}\right)$ be its jacobian matrix. We have

$$
\operatorname{Jac}\left(T^{\prime}\right)=\left(\begin{array}{ccccc}
M & 0 & 0 & 0 & 0 \\
* & x_{n-1} & x_{n} & 0 & 0 \\
* & 0 & 0 & 2 M x_{n-1} & 2 M x_{n}
\end{array}\right) .
$$

We can conclude in an obvious way. Similarly we can prove that $\{F=$ $0\} \cap\left\{\tilde{g_{2}}=0\right\} \cap\left\{M \tilde{g}_{1}+x_{n-1} \frac{\partial\left(\tilde{g}_{1}, F\right)}{\partial\left(x^{\prime}, x_{n-1}\right)}+x_{n} \frac{\partial\left(\tilde{g}_{1}, F\right)}{\partial\left(x^{\prime}, x_{n}\right)}=0\right\}$ and $\{F=$ $0\} \cap\left\{M \tilde{g}_{1}+x_{n-1} \frac{\partial\left(\tilde{g}_{1}, F\right)}{\partial\left(x^{\prime}, x_{n-1}\right)}+x_{n} \frac{\partial\left(\tilde{g}_{1}, F\right)}{\partial\left(x^{\prime}, x_{n}\right)}=0\right\} \cap\left\{M \tilde{g}_{2}+x_{n-1} \frac{\partial\left(\tilde{g}_{2}, F\right)}{\partial\left(x^{\prime}, x_{n-1}\right)}+\right.$ $\left.x_{n} \frac{\partial\left(\tilde{g}_{2}, F\right)}{\partial\left(x^{\prime}, x_{n}\right)}=0\right\}$ are finite sets outside $\left\{M_{\mathbf{C}}=0\right\}$. Thus we have shown that for almost all $\left(\alpha_{n-1}, \alpha_{n}, \beta_{n-1}, \beta_{n}\right), \tilde{g_{1}} \tilde{g}_{2 \mid W_{\mathbf{C}}}$ admits a finite set of critical points outside $\left\{M_{\mathbf{C}}=0\right\}$.

It remains to prove the "genericity" for the entire manifold $W_{\mathbf{C}}$. We still have two polynomials $g_{1}, g_{2}: \mathbf{R}^{n} \rightarrow \mathbf{R}$. For each pair of $(n-2)$ tuples $\alpha^{\prime}=\left(\alpha_{1}, \ldots, \alpha_{n-2}\right)$ and $\beta^{\prime}=\left(\beta_{1}, \ldots, \beta_{n-2}\right)$, let us consider the two polynomials

$$
\begin{aligned}
& g_{1,\left(\alpha^{\prime}, 0,0\right)}=g_{1}+\alpha_{1} x_{1}+\ldots \alpha_{n-2} x_{n-2}, \\
& g_{2,\left(\beta^{\prime}, 0,0\right)}=g_{2}+\beta_{1} x_{1}+\ldots \beta_{n-2} x_{n-2} .
\end{aligned}
$$

The previous study implies that for almost all $\left(\alpha_{n-1}, \alpha_{n}, \beta_{n-1}, \beta_{n}\right) \in$ $\mathbf{R}^{4}$ the function $g_{1,\left(\alpha^{\prime}, \alpha_{n-1}, \alpha_{n}\right)} \times g_{2,\left(\beta^{\prime}, \beta_{n-1}, \beta_{n}\right)}$ admits a finite number of critical points in $W_{\mathbf{C}} \backslash\left\{M_{\mathbf{C}}=0\right\}$ where

$$
g_{1,\left(\alpha^{\prime}, \alpha_{n-1}, \alpha_{n}\right)}=g_{1,\left(\alpha^{\prime}, 0,0\right)}+\alpha_{n-1} x_{n-1}+\alpha_{n} x_{n},
$$




$$
g_{2,\left(\beta^{\prime}, \beta_{n-1}, \beta_{n}\right)}=g_{2,\left(\beta^{\prime}, 0,0\right)}+\beta_{n-1} x_{n-1}+\beta_{n} x_{n} .
$$

Now let $S_{n-1, n}$ be the set of points $(\alpha, \beta) \in \mathbf{R}^{n} \times \mathbf{R}^{n}$ such that $g_{1, \alpha} \times$ $g_{2, \beta} \mid W_{\mathbf{C}}$ does not admit a finite number of critical points in $W_{\mathbf{C}} \backslash\left\{M_{\mathbf{C}}=\right.$ $0\}$. We have shown that each "horizontal slice" $S_{n-1, n} \cap\left\{\alpha^{\prime}\right\} \times \mathbf{R}^{2} \times$ $\left\{\beta^{\prime}\right\} \times \mathbf{R}^{2}$ has measure zero. By Fubini's theorem, $S_{n-1, n}$ has measure zero in $\mathbf{R}^{n} \times \mathbf{R}^{n}$. Now $W_{\mathbf{C}}$ can be covered by all open sets

$$
U_{i_{1}, \ldots, i_{n-2}}=W_{\mathbf{C}} \backslash\left\{\frac{\partial\left(F_{1}, \ldots, F_{n-2}\right)}{\partial\left(x_{i_{1}}, \ldots, x_{i_{n-2}}\right)}=0\right\} .
$$

Since these open sets are in a finite number, by the above study, for almost all $(\alpha, \beta) \in \mathbf{R}^{n} \times \mathbf{R}^{n}, g_{1, \alpha} \times g_{2, \beta \mid W_{\mathbf{C}}}$ admits a finite number of critical points in each $U_{i_{1}, \ldots, i_{n-2}}$, which implies that for almost all $(\alpha, \beta) \in \mathbf{R}^{n} \times \mathbf{R}^{n}, g_{1, \alpha} \times g_{2, \beta} W_{\mathbf{C}}$ has a finite number of critical points. This is equivalent to the finitude of the algebra

$$
\frac{\mathbf{R}[x]}{\left(F_{1}, \ldots, F_{n-2}, \frac{\partial\left(\tilde{g}_{1} \tilde{g}_{2}, F_{1}, \ldots, F_{n-2}\right)}{\partial\left(x_{i_{1}}, \ldots, x_{i_{n-1}}\right)}\right)},
$$

where $\tilde{g_{1}}=g_{1, \alpha}$ and $\tilde{g_{2}}=g_{2, \beta}$.

\section{References}

[AVG] ARNOLD, V.I., VARCHENKO, A.N., GUSEIN-ZADE, S.M. : Singularities of differentiable maps, (vol.1) Birkhauser (1988).

[BCRS] BECKER, E., CARDINAL, J.P., ROY, M.F., SZAFRANIEC, S.: Multivariate Bezoutians, Kronecker symbol and Eisenbud \& Levine formula, Algorithms in Algebraic Geometry and Applications Progress in Mathematics 143, 79-104 Birkhauser (1996).

[BR] BENEDETTI, R., RISLER, J.J. : Real algebraic and semi-algebraic sets, Hermann (1990).

[BCR] BOCHNAK, J., COSTE, M., ROY, M.F. : Géométrie algébrique réelle, Ergebnisse der Mathematik 12, Springer-Verlag (1987).

[Ca] CARDINAL, J.P. : Dualité et algorithmes itératifs pour le résolution des systèmes polynomiaux, Thèse, Université de Rennes 1 (1993).

[Dut1] DUTERTRE, N. : An algebraic formula for the Euler characteristic of some semi-algebraic sets, Journal of Pure and Applied Algebra 139, 41-60 (1999). 
[Dut2] DUTERTRE, N. : Sur la fibre d'un polynôme de $\mathbf{R}^{n}$ à points critiques isolés, Manuscripta Mathematica 100 437-454 (1999).

[Dut3] DUTERTRE, N. : About the Milnor fiber of a real map-germ, Prépublication de l'Institut de Recherche Mathématique de Rennes 99-04 (1999).

[Ei] EISENBUD, D. : An algebraic approach to the topological degree of a smooth map, Bull. Amer. Math. Soc. 84, 751-764 (1978).

[EL] EISENBUD, D., LEVINE, H.I. : An algebraic formula for the degree of a $C^{\infty}$ map-germ, Annals of Mathematics 106, 19-44 (1977).

[GRRT] GONZALEZ-VEGA, L., ROUILLIER, F., ROY, M.F., TRUJILLO, G. : Symbolic Recipes for Polynomials System Solving in Some Tapas of Computer Algebra, Springer-Verlag (ed. Cohen, Cuypers and Sterk) (1999).

[HL] HAMM, H., LE DUNG TRANG : Un théorème de Zariski du type de Lefschetz, Ann. Sci. Ecol. Norm. Sup. (3) 6, 317-355 (1973).

[Ku] KUNZ, E. : Kahler Differentials, Advanced Lectures in Mathematics, Braunschweig, Wuesbaden : Vieweg (1986).

[Mi] MILnOR, J. : Morse theory, Ann. Math. Stud. 51, Princeton University Press (1963).

[PRS] PEDERSEN, P., ROY M.F., SZPIRGLAS A. : Counting real zeroes in the multivariate case, Computational algebraic geometry, Eyssette et Galligo, eds. Prog. Math. 109, 203-224, Birkhäuser, Boston 1993.

[Ro] ROY, M.F. : Basic algorithms in real algebraic geometry and their complexity: from Sturm's theorem to the existential theory of reals, Lectures in Real Geometry, Berlin : de Gruyter, De Gruyter Expo. Math. 23 1-67 (1996).

[SS] SCHEJA, G., STORCH, U. : Uber Spurfinktionen bei vollstandingen Durschnitten, Journal reine angew Math 278/279, 174-190 (1975).

[Sz1] SZAFRANIEC, Z. : The Euler characteristic of algebraic complete intersections, Jour. reine angew Math. 397, 194-201 (1989).

[Sz2] SZAFRANIEC, Z. : A formula for the Euler characteristic of a real algebraic manifold, manuscripta mathematica 85, 345-360 (1994).

[Sz3] SZAFRANIEC, Z. : Topological degree and quadratic forms, Journal of Pure and Applied Algebra 141, 299-314 (1999). 
IMR, Université de Rennes 1

Campus de Beaulieu 35042 Rennes

(FRANCE)

E-mail: dutertre@maths.univ-rennes1.fr

Recibido: 20 de Febrero de 2000

Revisado: 24 de Octubre de 2000 